\title{
APPROXIMATION OF INTEGRAL FRACTIONAL LAPLACIAN AND FRACTIONAL PDES VIA SINC-BASIS
}

\author{
HARBIR ANTIL, PATRICK DONDL, AND LUDWIG STRIET
}

\begin{abstract}
Fueled by many applications in random processes, imaging science, geophysics, etc., fractional Laplacians have recently received significant attention. The key driving force behind the success of this operator is its ability to capture non-local effects while enforcing less smoothness on functions. In this article, we introduce a spectral method to approximate this operator employing a sinc basis. Using our scheme, the evaluation of the operator and its application onto a vector has complexity of $\mathcal{O}(N \log (N))$ where $N$ is the number of unknowns. Thus, using iterative methods such as Conjugate Gradient (CG), we provide an efficient strategy to solve fractional partial differential equations with exterior Dirichlet conditions on arbitrary Lipschitz domains. Our implementation works in both $2 d$ and $3 d$. We also recover the FEM rates of convergence on benchmark problems. For fractional exponent $s=1 / 4$ our current 3d implementation can solve the Dirichlet problem with $5 \cdot 10^{6}$ unknowns in under 2 hours on a standard office workstation. We further illustrate the efficiency of our approach by applying it to fractional Allen-Cahn and image denoising problems.
\end{abstract}

\section{INTRODUCTION}

This article is concerned with the numerical treatment of equations of the form

$$
\begin{aligned}
(-\Delta)^{s} u=f & \text { in } \Omega \\
u=0 & \text { in } \mathbb{R}^{d} \backslash \Omega,
\end{aligned}
$$

on an open bounded domain $\Omega \subset \mathbb{R}^{d}$ with Lipschitz boundary $\partial \Omega$. Furthermore, we consider applications to phase field models and imaging science.

A standard way to define the fractional Laplacian is via a principal value integral on functions in the Schwartz space $\mathcal{S}\left(\mathbb{R}^{d}\right)$ of rapidly decaying functions.

Definition 1.1. Let $u \in \mathcal{S}\left(\mathbb{R}^{d}\right)$ and $s \in(0,1)$. The operator $(-\Delta)^{s}$ is defined as

$$
(-\Delta)^{s} u(x)=C(d, s) P . V . \int_{\mathbb{R}^{d}} \frac{u(x)-u(y)}{|x-y|^{d+2 s}} d y \text { where } C(d, s)=\frac{s 2^{2 s} \Gamma(s+d / 2)}{\pi^{d / 2} \Gamma(1-s)}
$$

is a normalization constant.

This definition clearly shows the non-local character of the operator $(-\Delta)^{s}$. In order to evaluate $(-\Delta)^{s} u(x)$ at a single point $x \in \mathbb{R}^{d}$, one has to evaluate the singular integral over the full space $\mathbb{R}^{d}$. Furthermore, this fractional Laplacian only makes sense for functions which are defined on all of $\mathbb{R}^{d}$. The definition can be interpreted in a weak sense for regular distributions with suitable growth

LS gratefully acknowledges a doctoral scholarship from the Friedrich-Ebert-Stiftung. HA is partially supported by NSF grants DMS-1818772, DMS-1913004, the Air Force Office of Scientific Research (AFOSR) under Award NO: FA9550-19-1-0036, and Department of Navy, Naval PostGraduate School under Award NO: N00244-20-1-0005. 
conditions via integration by parts [Sil05, Chapter 2]. We will from now on use this interpretation implicitly when needed.

For the remainder of this paper, we will refer to problems of the form eq. (1.1) as the Dirichlet problem for the fractional Laplacian, or - more explicitly - as the fractional Poisson problem with Dirichlet exterior conditions. The fractional Laplacian of a function $u$ with support in $\Omega \subset \mathbb{R}^{d}$, $\Omega$ bounded, or, equivalently, of a function $u: \Omega \rightarrow \mathbb{R}$ which is extended by zero outside $\Omega$ will be denoted as the Dirichlet fractional Laplacian. We remark that other options to give meaning to fractional operators applied to functions defined on bounded domains exist (for an overview, see $\left[\mathrm{LPG}^{+} 20\right]$ ), but here, we will be concerned with the Dirichlet problem as described above.

On the entire $\mathbb{R}^{d}$, there are at least nine further, equivalent ways to define the fractional Laplacian, see [Kwa15]. A particularly useful one is the one defined using Fourier transform. See for example [DNPV12] for a proof of the following

Theorem 1.1. Let $s \in(0,1)$ and let $(-\Delta)^{s}: \mathcal{S}\left(\mathbb{R}^{d}\right) \rightarrow L^{2}\left(\mathbb{R}^{d}\right)$ the fractional Laplacian from definition 1.1. Then, for $u \in \mathcal{S}$,

$$
(-\Delta)^{s} u=\mathcal{F}^{-1}\left(|\omega|^{2 s}(\mathcal{F} u)\right)
$$

In principle, eq. (1.3) gives a direct method to solve eq. (1.1) via the Fourier transformation. This is, of course, numerically intractable as it is not possible to perform a discrete Fourier transformation (DFT) on an infinite domain. On the other hand, if one truncates the domain on which the Fourier transformation is performed, one instead obtains the fractional Laplacian of a function $u$ that was periodically extended outside the truncation. We note that this is a different operator than the Dirichlet fractional Laplacian that we will call periodic fractional Laplacian, denoted $(\overline{-\Delta})^{s}$.

Fractional partial differential equations (PDEs) of type eq. (1.1) have recently received a significant amount of attention. The interest in fractional operators of type $(-\Delta)^{s}$ with $s \in(0,1)$ stems from two facts: (i) these operators impose less smoothness (cf. the classical case $s=1$ ); (ii) even more importantly, these operators easily enable nonlocal interaction, recall that the classical derivatives lead to local operators.

The fractional Laplacian has been used as a regularizer in imaging [AB17b, ADK20]. Additionally, it can be derived using the so called long jump random walk [Val09]. Fractional diffusion-reaction equations such as fractional Allen-Cahn have been studied in [ASS15, ABS94, SV12, AB17b], and a fractional Cahn-Hilliard equation has been studied e.g. in [AM17, ASS15, AB17b]. A related example where, instead of the space-derivative the time-derivative is taken to fractional order in a diffusion-reaction equation is e.g. [JLLZ15]. Finally, the fractional Helmholtz equation has been recently derived in [WvBWA20] using the first principle arguments in conjunction with a constitutive relation. We emphasize that the fractional Laplacian in [WvBWA20] is of the so-called spectral type. From an optimal control point of view, fractional operators provide a great deal of flexibility, since the condition $u=0$ is imposed in the exterior $\mathbb{R}^{d} \backslash \Omega$ of the domain $\Omega$. Therefore it has been possible to introduce a new type of optimal control (called Exterior Control) using fractional PDEs [AKW19].

It has been noted in [CS06] that - in $\mathbb{R}^{d}$ - the definitions eq. (1.2) and eq. (1.3) are further equivalent to the so called extension problem. However, even for functions $u$ such that $\operatorname{supp} u \subset \Omega$, with $\Omega$ bounded, the extension has to be performed on $\mathbb{R}^{d} \times(0, \infty)$ to compute the fractional Laplacian for the Dirichlet problem. It is still possible to define an extension problem on $\Omega \times(0, \infty)$ which is equivalent to the so-called spectral fractional Laplacian [ST10]. Using such an extension, 
efficient finite element based numerical methods have been proposed in [NOS15, MPSV18]. For completeness, we also refer to [AR19] for an extension problem where the fractional exponent $s$ is a function of the spatial variable $x \in \Omega$.

On the other hand, the numerical methods for problems of the type eq. (1.1) present even more challenges, as one needs to resolve the singular integrals. The first work that rigorously tackles numerics for eq. (1.1) using finite element method is by Acosta and Borthagaray [AB17a], see also [AG18]. However, the implementations in these works have been limited to $d=2$ dimensions. We also refer to another finite element approach of Bonito, Lei, and Pasciak [BLP19], which also works in $d=3\left[\mathrm{BBN}^{+} 18\right]$.

In contrast, we provide a spectral method to approximate the Dirichlet fractional Laplacian eq. (1.2) where we attempt to combine fast Fourier transform (FFT) efficiency with the ability to treat exterior value problems as in eq. (1.1). As we shall illustrate with numerical examples, this approach directly applies to the case when $d=3$, and extension to even higher dimensions is possible given efficient FFT implementations. Using our method, the application of the fractional operator has the same numerical complexity as a Fourier transform. To solve the Dirichlet problem eq. (1.1) we then use this operator, restricted to $\Omega$, within a conjugate gradient algorithm.

Related strategies have been suggested recently by other authors. Duo and Zhang introduced a finite difference scheme to efficiently solve equations involving the fractional Laplace operator [DZ19]. Their method relies on a finite difference approximation of the operator and the fact that this can be expressed as a matrix consisting of blocks of symmetric Toeplitz-Matrices which can be applied efficiently using discrete Fourier transformation based methods. Given this operator, they solve fractional PDEs using iterative methods. Minden and Ying introduced a method to discretize the integral operator eq. (1.2) which also leads to a Toeplitz-Matrix. Along with a preconditioner, they also use the conjugate gradient method to solve the arising systems for the fractional Dirichlet problem and fractional diffusion equations [MY20]. Another example for a spectral method was provided recently by $\mathrm{Xu}$ and Darve. They use eigenfunctions and eigenvalues w.r.t a specific weight of the Dirichlet fractional Laplacian to solve the fractional Dirichlet problem on the unit ball [XD18].

Recently, mesh-free methods based on radial basis functions (RBFs) to estimate fractional Laplacians [BWZ20, RRD19] have been proposed. Those methods are related to our method in the sense that they also rely on the element-wise application of an operator to basis functions. Further, they use the fact that the Fourier transformation of smooth functions has rapid decay. A novelty that our method shares with [BWZ20] is that we can treat classical and fractional PDE in a single framework, as illustrated in the experiments in the experiments in section 4.2.

A more general, unified framework for finite difference schemes to evaluate Fractional Laplacians for $d=1$ is presented by Huang and Oberman in [HO16]. In particular, they use a finite difference scheme based on sinc-interpolation and show an equivalence to a Fourier scheme on an inifinite interval. We extend those results to multiple dimensions and present numerical schemes to calculate the resulting integrals.

The article is organized as follows. In section 2 we briefly discuss some properties of the sincinterpolation used in the present work as well as a discrete version of the fractional Laplacian based on this interpolation. The numerical methods to efficiently evaluate fractional Laplacians, as well as some equivalences between scaled periodic and our discretized operators, are presented in section 3 . In a supplementary document, we provide more details on the implementation of our algorithms and on the computational complexity, see appendix A. Section 4 is devoted to validation of our 
theoretical results using various numerical examples in both $d=2$ and $d=3$. The $L^{2}$-convergence rates for finite element methods as stated in [AG18] are obtained also using our method. We conclude the article by successfully applying the proposed scheme to the fractional Allen-Cahn equation and image denoising problems.

\section{The SinC-Fractional LAPLACian}

In this section, we define our discrete approximation of the Dirichlet fractional Laplacian. First, in section 2.1, we introduce an appropriate discretization method for functions with bounded support. Following this, we use a discrete convolution to define our operator in section 2.2 .

2.1. sinc-Interpolation. It is a well-known fact that, for example, smooth functions with compact support on $\mathbb{R}$ can be well approximated by a weighted sum of scaled and shifted sinc-functions. The sinc-function is defined as

$$
\operatorname{sinc}(x)=\frac{\sin (\pi x)}{\pi x}
$$

Two different approaches of approximating functions with sinc-functions are found in the literature. The first approach is to consider the sinc-function as a wavelet scaling function and then to approximate in the sense of wavelets which leads to the so called Shannon-wavelets. A good overview on such techniques can be found in [Cat08]. They have also been used to approximate fractional derivatives as shown in [Cat15, Cat18].

The second approach, which we will pursue here, is to approximate a function $u: \mathbb{R} \rightarrow \mathbb{R}$ by

$$
u(x) \approx \sum_{k=-\infty}^{\infty} u(k h) \operatorname{sinc}\left(\frac{x-k h}{h}\right) .
$$

The sum on the right hand side (if it converges) is called Whittaker Cardinal Function, see, e.g., [MSW71] for more details. This approximation is rather precise [Ste10] and has found numerous applications, e.g., for solving ordinary differential equations, partial differential equations and integral equations [Ste00, Ste10], but also for approximating integrals arising in fractional calculus [BS11]. Furthermore, this is the method chosen in [HO16] to derive the weights for a finite difference scheme to compute fractional Laplacians in one dimension. Another way the sinc-functions are employed in the area of fractional PDEs is to use sinc-quadrature to compute the Dunford-Taylor integral arising from the fractional Laplacian, as done, e.g., in [BLP19].

In this work, we will use approximations similar to eq. (2.2), extended to multiple dimensions. For $x=\left(x_{1}, \ldots, x_{d}\right)^{T} \in \mathbb{R}^{d}$, we set

$$
\varphi(x)=\prod_{j=1}^{d} \operatorname{sinc}\left(x_{j}\right)
$$

as the reference basis function and define its scaled and shifted version $\varphi_{k}^{N}: \mathbb{R}^{d} \rightarrow \mathbb{R}$ for $k=$ $\left(k_{1}, \ldots, k_{d}\right)^{T} \in \mathbb{Z}^{d}$ by a tensor product

$$
\varphi_{k}^{N}(x)=\prod_{j=1}^{d} \varphi\left(N x_{j}-k_{j}\right), \quad x=\left(x_{1}, \ldots, x_{d}\right)^{T} \in \mathbb{R}^{d}
$$


where $\mathbb{Z}^{d}$ is the $d$-dimensional integer lattice. For $u: \mathbb{R}^{d} \longrightarrow \mathbb{R}$, we obtain the sinc approximation

$$
u_{N}(x)=\sum_{k \in \mathbb{Z}^{d}} u_{k} \varphi_{k}^{N}(x) \quad \text { where } u_{k}=u\left(x_{k}\right), x_{k}=k / N
$$

Since we are interested in computing the Dirichlet fractional Laplacian, our functions have compact support, which we from now on assume to be contained in the unit cube $[0,1)^{d}$ (the generalization to other, larger domains is trivial; our assumption is merely for notational convenience). We therefore may truncate the series to

$$
u_{N}(x)=\sum_{k_{1}=0}^{N-1} \cdots \sum_{k_{d}=0}^{N-1} u_{k} \varphi_{k}^{N}(x)=: \sum_{k \in \mathcal{I}_{N}^{d}} u_{k} \varphi_{k}^{N}(x),
$$

where, if $\mathcal{I}_{N}:=\{0, \ldots, N-1\}$, then $\mathcal{I}_{N}^{d} \subset \mathbb{Z}^{d}$ indicates its $d$-fold Cartesian product. Later in this work, we will also use the set $\mathcal{I}_{N}^{\prime}=\{-N / 2, \ldots, N / 2-1\}$ and its Cartesian, product respectively.

It is a well-known fact that the basis function $\varphi$ can be obtained as the inverse Fourier transform of the indicator function of a square, i.e., for $D=[-\pi ; \pi]^{d}$ we have

$$
\left(\mathcal{F}^{-1} \chi_{D}(\omega)\right)(x)=(2 \pi)^{d} \varphi(x)
$$

where $\chi_{D}(x)=1$ if $x \in D, 0$ otherwise Similarly, $\varphi_{k}^{N}(x)$ can be obtained as

$$
\varphi_{k}^{N}(x)=\varphi(N x-k)=\mathcal{F}^{-1} \underbrace{\left((2 \pi N)^{-d} \chi_{D_{N}}(\omega) e^{-\mathrm{i} \omega k / N}\right)}_{=\mathcal{F} \varphi_{k}^{N}(\omega)}(x)
$$

where $D_{N}=[-N \pi ; N \pi]^{d} \subset \mathbb{R}^{d}$.

2.2. The discrete operator. Given a real function $u$ with supp $u \subset[0 ; 1)^{d}$, we want to apply the Dirichlet Fractional Laplace operator $(-\Delta)^{s}$ of eq. (1.3) to its sinc-approximation $u_{N}$. We shall write

$$
(-\Delta)_{N}^{s} u:=(-\Delta)^{s} u_{N}
$$

and call the operator $(-\Delta)_{N}^{s}$ the sinc-fractional Laplacian. We are restricted to grid points, so let $x_{\kappa}=\kappa / N, \kappa \in \mathcal{I}_{N}^{d}$. As $\mathcal{I}_{N}^{d}$ is finite and $(-\Delta)^{s}$ is linear, we obtain

$$
\begin{aligned}
(-\Delta)^{s} u_{N}\left(x_{\kappa}\right) & =(-\Delta)^{s}\left(\sum_{k \in \mathcal{I}_{N}^{d}} u_{k} \varphi_{k}^{N}\left(x_{\kappa}\right)\right) \\
& =\sum_{k \in \mathcal{I}_{N}^{d}} u_{k} \underbrace{\left((-\Delta)^{s} \varphi_{k}^{N}\right)\left(x_{\kappa}\right)}_{=: \Phi^{N}(\kappa-k)} \\
& =\sum_{k \in \mathcal{I}_{N}^{d}} u_{k} \Phi^{N}(\kappa-k) .
\end{aligned}
$$

where we have defined $\Phi^{N}(\kappa-k)=(-\Delta)^{s} \varphi_{k}^{N}\left(x_{\kappa}\right)$ for $k, \kappa \in \mathcal{I}_{N}^{d}$. In the remainder of this paper, we will occasionally use the notation $\Phi_{K}^{N}=\Phi^{N}(\kappa-k)$, with $K=\kappa-k$ when it is clear from the context. Notice that eq. (2.6) denotes a discrete convolution. The computation of this convolution is the application of the sinc-fractional Laplacian.

In other words, we obtain the sinc-fractional Laplacian $(-\Delta)^{s} u_{N}\left(x_{\kappa}\right)$ for any grid point $x_{\kappa}$ as the discrete convolution of $\mathbf{u}=\left(u_{k}\right)_{k \in \mathcal{I}_{N}^{d}}$ and $\Phi^{N}$. Such a convolution can be implemented efficiently 
using the FFT algorithm once $\Phi^{N}(\kappa-k)$ is known for all $k, \kappa \in \mathcal{I}_{N}^{d}$. More precisely, the circular discrete convolution of two vectors $\mathbf{x}, \mathbf{y} \in \mathbb{R}^{N^{d}}$ can be calculated as

$$
\begin{aligned}
\left(\mathbf{x} *_{d} \mathbf{y}\right)(k) & :=\sum_{\kappa \in \mathcal{I}_{N}^{d}} \overline{\mathbf{x}}(\kappa) \cdot \overline{\mathbf{y}}(k-\kappa) \\
& =\operatorname{IDFT}\{(\operatorname{DFT} \mathbf{x}) \circ(\operatorname{DFT} \mathbf{y})\}(k)
\end{aligned}
$$

where $\circ$ denotes the component-wise product of vectors, and DFT and IDFT denote the discrete Fourier transformation and the inverse discrete Fourier transformation, respectively. By circular we mean that negative components of indices $\kappa-k$ are mapped circularly to their positive counterparts, in formulas

$$
\overline{\mathbf{x}}(K)= \begin{cases}\mathbf{x}(K) & \text { if } K \geq 0 \\ \mathbf{x}(K+N) & \text { if } K<0\end{cases}
$$

While the evaluation of eq. (2.7) is of complexity $\mathcal{O}\left(\left(N^{d}\right)^{2}\right)$, if evaluated for each $k$, the simultaneous evaluation of eq. (2.8) for all $k$ can be implemented in $\mathcal{O}\left(N^{d} \log \left(N^{d}\right)\right)$ time.

For our application, we do not actually want to apply the circular convolution, but the convolution where we extend by zero instead of periodically. As we still want to use the FFT-based algorithm to evaluate eq. (2.6) because of its computational efficiency, we set

$$
\bar{u}_{k}= \begin{cases}u_{k} & \text { if } k \geq 0 \\ 0 & \text { otherwise }\end{cases}
$$

where the expression $k \geq 0$ is meant component-wise. Then, we have that

$$
(-\Delta)^{s} u_{N}\left(x_{\kappa}\right)=\left(\bar{u} *_{d} \Phi^{N}\right)(\kappa)
$$

which we implement using a FFT of size $(2 N)^{d}$. Further details on the implementation and pseudocode can be found in appendix A.

We have shown that we can obtain the basis function $\varphi(\cdot)$ as the inverse Fourier transformation of the indicator function of a square in $\mathbb{R}^{d}$. This can in principle be used to obtain the integral fractional Laplacian $\left((-\Delta)^{s} \varphi\right)$ of the basis functions, since for $x \in \mathbb{R}^{d}$, we have

$$
\begin{aligned}
\left((-\Delta)^{s} \varphi\right)(x) & =\mathcal{F}^{-1}\left(|\omega|^{2 s}(\mathcal{F} \varphi)\right) \\
& =(2 \pi)^{-d} \int_{D}|\omega|^{2 s} e^{\mathrm{i} \omega \cdot x} d \omega,
\end{aligned}
$$

and

$$
\begin{aligned}
\Phi^{N}(\kappa-k) & =(2 \pi N)^{-d} \int_{D_{N}}|\omega|^{2 s} e^{\mathrm{i} \omega \cdot\left(x_{\kappa}-k / N\right)} d \omega \\
& =(2 \pi)^{-d} N^{2 s} \int_{D}|\omega|^{2 s} e^{\mathrm{i} \omega \cdot(\kappa-k)} d \omega \\
& =N^{2 s}\left((-\Delta)^{s} \varphi\right)(\kappa-k) .
\end{aligned}
$$

However, using this equality directly is impractical as we would have to evaluate the oscillating integral for each multi-index $k$. For the one dimensional case, it is possible to circumvent this issue through the use of the confluent hypergeometric function [HO16], but for $d>1$ a numerical solution must be found. Clearly, calculating $\Phi^{N}$ itself is not necessary in order to implement eq. (2.6) as we only need the discrete Fourier transformation $\hat{\Phi}^{N}$ of $\Phi^{N}$. In section 3.3 we show how $\hat{\Phi}^{N}$ can be obtained efficiently. 


\section{Numerical Methods}

The goal of this section is to introduce our numerical methods. We begin with section 3.1 where we discuss the computation of fractional Laplacians using simple Fourier methods as mentioned in the introduction. In section 3.2, we show that the sinc-fractional Laplacian applied to a function with support in $[0,1)^{d}$, as defined in eq. (2.6), can be seen as a limit $S \rightarrow \infty$ of fractional Laplacians obtained by standard Fourier transforms of the function periodically extended outside $[0, S)^{d}$.

We then describe, in section 3.3, our numerical quadrature method used to compute $\hat{\Phi}$. The solution strategy to the Dirichlet exterior value problem eq. (1.1) is discussed in section 3.4.

3.1. Computation of the periodic fractional Laplacian. To experimentally test that our discrete approximation eq. (2.6) indeed approaches the Dirichlet fractional Laplacian, we can compare it to the periodic fractional Laplacian which is calculated by extending $u$ periodically outside of a truncation domain, instead of extending $u$ by 0 .

For periodic functions, the periodic fractional Laplacian and the integral fractional Laplacian are equal [AV19]. If the Dirichlet fractional Laplacian is approximated with the periodic fractional Laplacian on a finite domain, an error is introduced due to the implicit periodization of the function. This effect, however, is reduced if the function is scaled with a factor $S>1$ before the application of the periodic fractional Laplacian and rescaled to the original domain afterwards. Heuristically, this occurs because the additional support introduced by the periodic continuation becomes shifted further away from the original support. The error is then on the order of $S^{-(d+2 s)}$. This estimate is summarized in the following Lemma. The main motivation for this Lemma is the fact that in the next section we shall establish an equivalence between the scaled periodic fractional Laplacian and the sinc-fractional Laplacian, see theorems 3.1 and 3.2.

Lemma 3.1. Let $u \in L^{1}\left(\mathbb{R}^{d}\right)$ with $\operatorname{supp} u=\Omega \subset[0,1)^{d}$. Let $(-\Delta)^{s}$ be the Dirichlet fractional Laplacian (see equation eq. (1.2)) and $(\overline{-\Delta})^{s}$ be the periodic fractional Laplacian (applied on the function restricted to $\left.[0,1)^{d}\right)$. Assume furthermore that $(-\Delta)^{s} u \in L^{1}\left([0,1)^{d}\right)$. Then, for a.e. $x \in(0,1)^{d}, S$ sufficiently large, we have

$$
S^{-2 s}\left(\widetilde{(-\Delta)^{s}} u(S \cdot)\right)(x / S)=(-\Delta)^{s} u(x)+\mathcal{O}\left(S^{-(d+2 s)}\right) .
$$

Proof. Assume first that $u \in C_{c}^{\infty}\left([0,1)^{d}\right)$, fix $x \in(0,1)^{d}$ and set $\tilde{x}=x / S, u_{S}=u(S \cdot)$. We calculate

$$
\begin{aligned}
(\widetilde{(-\Delta})^{s} u_{S}(\tilde{x})= & (-\Delta)^{s}\left(\sum_{k \in \mathbb{Z}^{d}} u_{S}(\cdot-k)\right)(\tilde{x}) \\
= & C(d, s) \int_{\mathbb{R}^{d}} \frac{u(S \tilde{x})-u(S y)}{|\tilde{x}-y|^{d+2 s}} d y \\
& +C(d, s) \sum_{k \in \mathbb{Z}^{d} \backslash\{0\}} \int_{\mathbb{R}^{d}} \frac{u(S \tilde{x}-S k)-u(S y-S k)}{|\tilde{x}-y|^{d+2 s}} d y,
\end{aligned}
$$

where we note that the sum in equation (3.3) converges absolutely, owing to (3.4) and the estimate (3.5) below. 
A linear transformation in the term in (3.2) yields

$$
C(d, s) \int_{\mathbb{R}^{d}} \frac{u(S \tilde{x})-u(S y)}{|\tilde{x}-y|^{d+2 s}} d y=C(d, s) \int_{\mathbb{R}^{d}} \frac{u(S \cdot x / S)-u(S y)}{|x / S-y|^{d+2 s}} \frac{S^{d}}{S^{d}} d y=S^{2 s}(-\Delta)^{s} u(x) .
$$

For the integral in the summand in (3.3), we have for $k \neq 0$

$$
\int_{\mathbb{R}^{d}} \frac{[u(S \tilde{x}-S k)-u(S y-S k)]}{|\tilde{x}-y|^{d+2 s}} d y=-\int_{\mathbb{R}^{d}} \frac{u(S y-S k)}{|\tilde{x}-y|^{d+2 s}} d y
$$

as $S \tilde{x}-S k \notin(0,1)^{d} \supset \operatorname{supp} u$. Furthermore,

$$
\left|\int_{\mathbb{R}^{d}} \frac{u(S y-S k)}{|\tilde{x}-y|^{d+2 s}} d y\right|=S^{2 s} \int_{(0,1)^{d}} \frac{u(y)}{|x-y-S k|^{d+2 s}} d y \leq\|u\|_{L^{1}\left((0,1)^{d}\right)} \cdot C^{\prime} \frac{1}{S^{d}} \frac{1}{|k|^{d+2 s}},
$$

where the constant $C^{\prime}$ remains bounded for large $S$. Summing over $k \neq 0$ and dividing by $S^{2 s}$ yields the result.

For $(-\Delta)^{s} u \in L^{1}$ only, note that we still have $(\widetilde{(-\Delta})^{s} u_{S}(S \cdot)=S^{2 s}(-\Delta)^{s} u(\cdot)-\sum_{k \in \mathbb{Z}^{d} \backslash\{0\}} \int_{\mathbb{R}^{d}} \frac{u(S y-S k)}{|S \cdot-y|^{d+2 s}} d y$ and that the second term is a non-singular integral that can be estimated as above.

The periodic fractional Laplacian can be discretized using the DFT. A comprehensive overview is provided in $[\mathrm{AB} 17 \mathrm{~b}]$. Briefly repeated, the $N^{d}$-point discrete Fourier transformation of a vector $\mathbf{x} \in \mathbb{R}^{N^{d}}$ is defined as

$$
\left(\mathrm{DFT}_{N} \mathbf{x}\right)_{k}=\hat{x}_{k}=\sum_{j_{1}=0}^{N-1} \cdots \sum_{j_{d}=0}^{N-1} x_{j} \mathrm{e}^{-\mathrm{i} \frac{2 \pi j \cdot k}{N}}
$$

and the inverse discrete Fourier transform is

$$
\left(\operatorname{IDFT}_{N} \hat{\mathbf{x}}\right)_{k}=x_{k}=\frac{1}{N^{d}} \sum_{j_{1}=0}^{N-1} \cdots \sum_{j_{d}=0}^{N-1} \hat{x}_{j} \mathrm{e}^{\mathrm{i} \frac{2 \pi j \cdot k}{N}} .
$$

If the size $N$ of the DFT is obvious, we will omit the subscript index $N$. The $N^{d}$-point discrete periodic fractional Laplacian of $u:[0 ; 1)^{d} \longrightarrow \mathbb{R}$ is calculated via

$$
\left.(\widetilde{(\overline{-\Delta}})_{N}^{s} u\right)\left(x_{\kappa}\right)=\left(\operatorname{IDFT}_{N}\left(\zeta \circ \operatorname{DFT}_{N} u\right)\right)(\kappa)
$$

where $\circ$ denotes the Hadamard (entrywise) product, DFT and IDFT the $N^{d}$-point discrete (inverse) Fourier transformation and

$$
\zeta_{k}=|2 \pi k|^{2 s} \text {. }
$$

Note that usual FFT implementations of the DFT calculate the discrete spectrum of $f$ in the range $\{0, \ldots, N-1\}^{d}$. To have the factors $\zeta$ at the correct scale, one has to shift the Fourier coefficients periodically to the interval $\{-N / 2, \ldots, N / 2-1\}$ to obtain

$$
\begin{aligned}
& \left((\widetilde{(-\Delta})_{N}^{s} u\right)\left(x_{\kappa}\right)=\left(\operatorname{IDFT}_{N}\left(\zeta \circ \operatorname{DFT}_{N} u\right)\right)(\kappa) \\
& =\frac{1}{N^{d}} \sum_{k_{1}=-N / 2}^{N / 2-1} \cdots \sum_{k_{d}=-N / 2}^{N / 2-1}|2 \pi k|^{2 s} \hat{u}_{k} \mathrm{e}^{i \frac{2 \pi k \cdot \kappa}{N}} .
\end{aligned}
$$


If $S \in \mathbb{N}$, we calculate the discretized scaled periodic fractional Laplacian in eq. (3.1) similarly using the $S N$-point DFT and inverse DFT as follows: we extend the vector

$$
\mathbf{u}=\left(u_{k}\right)_{k \in \mathcal{I}_{N}^{d}} \in \mathbb{R}^{N^{d}}, u_{k}=u(k / N)
$$

to a vector

$$
\overline{\mathbf{u}}=\left(\bar{u}_{k}\right)_{k \in \mathcal{I}_{S N}^{d}} \in \mathbb{R}^{(S N)^{d}}, \bar{u}_{k}= \begin{cases}u_{k} & \text { if } k<N \\ 0 & \text { otherwise }\end{cases}
$$

where the <-sign is meant component-wise and obtain

$$
\begin{aligned}
\left((\widetilde{-\Delta})_{S, N}^{s} u\right)\left(x_{\kappa}\right) & =\left(\operatorname{IDFT}_{S N}\left(\zeta \circ \operatorname{DFT}_{S N} u\right)\right)(\kappa) \\
& =\frac{1}{(S N)^{d}} \sum_{j_{1}=-S N / 2}^{S N / 2-1} \cdots \sum_{j_{d}=-S N / 2}^{S N / 2-1}|2 \pi j|^{2 s} \hat{u}_{j} \mathrm{e}^{\mathrm{i} \frac{2 \pi j \cdot \kappa}{S N}} \\
& =\frac{(2 \pi)^{2 s}}{(S N)^{d}} \sum_{j \in \mathcal{I}_{S N}^{d}}|j|^{2 s}\left(\sum_{k \in \mathcal{I}_{S N}^{d}} u_{k} \mathrm{e}^{-\mathrm{i} \frac{2 \pi j \cdot k}{S N}}\right) \mathrm{e}^{\mathrm{i} \frac{2 \pi j \cdot \kappa}{S N}} \\
& =\frac{(2 \pi)^{2 s}}{(S N)^{d}} \sum_{k \in \mathcal{I}_{N}^{d}} u_{k} \sum_{j \in \mathcal{I}_{S N}^{\prime}}|j|^{2 s} \mathrm{e}^{\mathrm{i} \frac{2 \pi}{S N} j \cdot(\kappa-k)} .
\end{aligned}
$$

The last line is certainly not the most efficient way to evaluate $\left.(\widetilde{(-\Delta})_{S, N}^{s} u\right)\left(x_{\kappa}\right)$ - this should instead be done via the FFT algorithm as stated in the first line. However, the expression will be needed in the following to show an equivalence of the sinc-fractional Laplacian and the scaled periodic fractional Laplacian.

\subsection{Equivalence of the scaled periodic fractional Laplacian and the sinc-fractional} Laplacian. There is a certain equivalence of the discrete scaled periodic fractional Laplacian and the sinc-fractional Laplacian. Precisely, if the integration in the calculation of $\Phi$, see eq. (2.10) is done exactly, the $N^{d}$-point sinc-fractional Laplacian is the same as the $N^{d}$-point discrete scaled periodic fractional Laplacian with infinite scale factor. To be precise, we have the following theorem.

Theorem 3.1. Let $u \in C_{c}\left([0 ; 1]^{d}\right)$. Then, for $S \longrightarrow \infty$

$$
S^{-2 s}\left((\widetilde{-\Delta})_{S, N}^{s} u\right)\left(x_{\kappa}\right) \longrightarrow\left((-\Delta)_{N}^{s} u\right)\left(x_{\kappa}\right) \quad \forall x_{\kappa}=\kappa / N, \kappa \in \mathcal{I}_{N}^{d} .
$$

Proof. Refer to equations (3.11), (2.6) to see that it is enough to show that

$$
S^{-2 s} \frac{(2 \pi)^{2 s}}{(S N)^{d}} \sum_{j \in \mathcal{I}_{S N}^{\prime d}}|j|^{2 s} \mathrm{e}^{\mathrm{i} \frac{2 \pi}{S N} j \cdot(\kappa-k)} \longrightarrow \Phi^{N}(\kappa-k)
$$

$\forall K:=\kappa-k$ as $S \longrightarrow \infty$. Indeed, we have

$$
\Phi^{N}(\kappa-k)=(2 \pi N)^{-d} \int_{D_{N}}|\omega|^{2 s} \mathrm{e}^{\mathrm{i} \frac{\omega \cdot(\kappa-k)}{N}} d \omega
$$


(see equation (2.10)) and for the left-hand side of (3.12), we have

$$
\begin{aligned}
& \frac{(2 \pi)^{2 s}}{N^{d}} S^{-(d+2 s)} \sum_{j \in \mathcal{I}_{S N}^{\prime d}}|j|^{2 s} \mathrm{e}^{\mathrm{i} \frac{2 \pi}{S N} j \cdot K} \\
= & \frac{(2 \pi)^{2 s}}{N^{d}} S^{-(d+2 s)} \sum_{j \in \mathcal{I}_{N}^{\prime d}} \sum_{i \in \mathcal{I}_{S}^{d}}|S j+i|^{2 s} \mathrm{e}^{\mathrm{i} \frac{2 \pi}{S N}(S j+i) \cdot K} \\
= & \frac{(2 \pi)^{2 s}}{N^{d}} \sum_{j \in \mathcal{I}_{N}^{\prime \prime}} \sum_{i \in \mathcal{I}_{S}^{d}} S^{-d}|j+i / S|^{2 s} \mathrm{e}^{\mathrm{i} \frac{2 \pi}{N}(j+i / S) \cdot K} \\
\stackrel{S \rightarrow \infty}{\longrightarrow} & \frac{(2 \pi)^{2 s}}{N^{d}} \sum_{j \in \mathcal{I}_{N}^{\prime d}} \int_{j_{1}}^{j_{1}+1} \cdots \int_{j_{d}}^{j_{d}+1}|\omega|^{2 s} \mathrm{e}^{\mathrm{i} \frac{2 \pi}{N} \omega \cdot K} \\
= & \frac{(2 \pi)^{2 s}}{N^{d}} \int_{\left[-\frac{N}{2} ; \frac{N}{2}\right]^{d}}|\omega|^{2 s} \mathrm{e}^{\mathrm{i} \frac{2 \pi}{N} \omega \cdot K} d \omega \\
= & (2 \pi N)^{-d} \int_{D_{N}}|\omega|^{2 s} \mathrm{e}^{\mathrm{i} \frac{\omega \cdot K}{N}} d \omega
\end{aligned}
$$

which completes the proof.

A similar result is presented in [HO16] for functions with non-compact support using the semidiscrete Fourier transformation. In section 3.3, see theorem 3.2, we show a direct relation between simple quadrature rules to evaluate the discrete convolution kernel $\hat{\Phi}$ and scaled Fourier fractional Laplacians.

3.3. Setting up the convolution kernel. As stated before, we aim to calculate the discrete Fourier transformation $\hat{\Phi}$ of $\Phi$ directly, instead of having to calculate it as the DFT of $\Phi$ as the latter is hard to obtain. While the fast implementation of the convolution is standard and can be found in many textbooks, our contribution is the formulation that makes the use of fast convolution algorithms applicable. Therefore, let $\mathcal{I}^{\prime d}{ }_{2 N}=\{-N, \ldots,(N-1)\}^{d}$ and $k \in \mathcal{I}^{\prime d}{ }_{2 N}$ a multiindex. Let $\Phi_{k}=(-\Delta)^{s} \varphi_{N}\left(x_{k}\right), x_{k}=k / N \in \mathbb{R}^{(2 N)^{d}}$. Let $\hat{\Phi}=\operatorname{DFT}_{2 N}(\Phi)$ the discrete Fourier transform of $\Phi \in \mathbb{R}^{(2 N)^{d}}$. We start the computation with the fact that

$$
\begin{aligned}
\hat{\Phi}_{k} & =\sum_{j \in \mathcal{I}_{2 N}^{\prime d}} \Phi_{j} \mathrm{e}^{-\mathrm{i} 2 \pi k \cdot j /(2 N)} \\
& =N^{2 s}(2 \pi)^{-d} \sum_{j \in \mathcal{I}^{\prime d}{ }_{2 N}}\left(\int_{D}|\omega|^{2 s} \mathrm{e}^{\mathrm{i} \omega \cdot j} d \omega\right) \mathrm{e}^{-\mathrm{i} \pi k \cdot j / N} \\
& =N^{2 s}(2 \pi)^{-d} \int_{D}|\omega|^{2 s} \sum_{j \in \mathcal{I}_{2 N}^{\prime d}} \mathrm{e}^{\mathrm{i} j \cdot\left(\omega-\frac{\pi}{N} k\right)} d \omega
\end{aligned}
$$


where we use the definition of the discrete Fourier transformation in the first equation, see eq. (3.6) and the formula for $\Phi_{j}$, see eq. (2.10), in the second equation. For $x \in \mathbb{R}$, define

$$
Y(x):=\sum_{j=-N}^{N-1} \mathrm{e}^{\mathrm{i} j x}= \begin{cases}\frac{\mathrm{e}^{-\mathrm{i} N x}\left(\mathrm{e}^{2 \mathrm{i} N x}-1\right)}{\mathrm{e}^{\mathrm{e}^{x}-1}} & \text { if } \mathrm{e}^{\mathrm{i} x}-1 \neq 0 \\ 2 N & \text { otherwise }\end{cases}
$$

To simplify the sum in eq. (3.15), we observe that for $x \in \mathbb{R}^{d}$, we have

$$
\begin{aligned}
\sum_{j \in \mathcal{I}_{2 N}^{\prime d}} \mathrm{e}^{\mathrm{i} j \cdot x} & =\sum_{j_{1}=-N}^{N-1} \cdots \sum_{j_{d}=-N}^{N-1} \mathrm{e}^{\mathrm{i}\left(j_{1} x_{1}+\cdots+j_{d} x_{d}\right)} \\
& =\prod_{i=1}^{d} Y\left(x_{i}\right) \\
& =: Y_{d}(x)
\end{aligned}
$$

plug this into eq. (3.15) and obtain

$$
\begin{aligned}
\hat{\Phi}_{k} & =N^{2 s}(2 \pi)^{-d} \int_{D}|\omega|^{2 s} \sum_{j \in \mathcal{I}_{2 N}^{\prime d}} \mathrm{e}^{\mathrm{i} j \cdot(\omega-\pi / N k)} d \omega \\
& =N^{2 s}(2 \pi)^{-d} \int_{D}|\omega|^{2 s} Y_{d}(\omega-\pi / N k) d \omega \\
& =\underbrace{N^{2 s}(2 \pi)^{-d}\left(\frac{\pi}{N}\right)^{d+2 s}}_{=\pi^{2 s} \cdot(2 N)^{-d}} \int_{[-N ; N]^{d}}|\omega|^{2 s} Y_{d}\left(\frac{\pi}{N}(\omega-k)\right) d \omega .
\end{aligned}
$$

Now, we have the same domains for $k$ and $\omega$. Finally, we note that the second factor $Y_{d}(\cdots)$ in the integrand is periodic with the length $2 N$ of the integrals and, thus, eq. (3.15) can be implemented as a convolution using the FFT algorithm and using quadrature rules as follows:

$$
\begin{aligned}
\hat{\Phi}_{k} & =\underbrace{\pi^{2 s}(2 N)^{-d}}_{C(N, d, s)} \int_{[-N ; N]^{d}}|\omega|^{2 s} Y_{d}\left(\frac{\pi}{N}(\omega-k)\right) d \omega \\
& \approx C(N, d, s) \sum_{j \in \mathcal{I}_{2 N}^{\prime d}} \sum_{i=1}^{N_{Q}} \alpha_{i}\left|j+x_{i}\right|^{2 s} Y_{d}\left(\frac{\pi}{N}\left(j+x_{i}-k\right)\right) \\
& =C(N, d, s) \sum_{i=1}^{N_{Q}} \alpha_{i} \sum_{j \in \mathcal{I}_{2 N}^{\prime d}}\left|j+x_{i}\right|^{2 s} Y_{d}\left(-\frac{\pi}{N}(k-j)+\frac{\pi}{N} x_{i}\right) .
\end{aligned}
$$

Where $\left(x_{i}, \alpha_{i}\right)_{i=1, \ldots, N_{Q}}$ is a quadrature rule on $[0 ; 1]^{d}$. The inner sum can be obtained as a discrete convolution for each $i$ using two forward and one backward DFTs. In summary, we have to execute $3 N_{Q}$ DFTs of size $(2 N)^{d}$ to obtain $\hat{\Phi}$. This is the computationally most demanding step in our algorithm, but it has to be performed only once when $\hat{\Phi}$ is applied multiple times. The values of $\hat{\Phi}$ could even be stored for given of $N$ and $s$. It is also possible to reduce the sizes of the DFTs to $(2 N-1)^{d}$. This, however, complicates the preceding computations. 
A consequence of theorem 3.1 is that the exactness of the integration in eq. (3.22) is decisive for the accuray of our method. A trivial choice for the integration points $x_{i}$ and weights $\alpha_{i}$ is

$$
x_{i}=\frac{i}{N_{Q}}, i \in\left\{0, \ldots, N_{Q}-1\right\}^{d} \text { and } \alpha_{i}=\alpha=\frac{1}{N_{Q}^{d}} .
$$

For this quadrature rule, the sinc-fractional Laplacian is exactly the same as the discrete scaled periodic fractional Laplacian with $S=2 N_{Q}$. We will discuss more possibilities along with numerical experiments in section 4.1.

Theorem 3.2. If $\hat{\Phi}$ in eq. (3.22) is calculated using the quadrature rule from eq. (3.23), then with $S=2 N_{Q}$ we have for all $\kappa$ that

$$
\left.\left((-\Delta)_{N}^{S} u\right)\left(x_{\kappa}\right)=(\widetilde{(-\Delta})_{S, N}^{s} u\right)\left(x_{\kappa}\right)
$$

Proof. We already derived that

$$
\left((\widetilde{-\Delta})_{S, N}^{S} u\right)\left(x_{\kappa}\right)=\frac{(2 \pi)^{2 s}}{S^{2 s} N S^{d}} \sum_{k \in \mathcal{I}_{N}^{d}} u_{k} \underbrace{\sum_{j \in \mathcal{I}_{N S}^{d}}|j|^{2 s} \mathrm{e}^{\mathrm{i} \frac{2 \pi j(\kappa-k)}{S N}}}_{=\tilde{\Phi}(\kappa-k)},
$$

see 3.11 . Using 2.6 one can verify that it is enough to show that

$$
\Phi^{N}(\kappa-k)=\frac{(2 \pi)^{2 s}}{S^{2 s} N S^{d}} \tilde{\Phi}(\kappa-k)
$$

for an appropriate choice of $\left(\alpha_{i}, x_{i}\right)_{i=1, \ldots, N_{Q}}$. From 3.22, we derive using 3.23 that

$$
\hat{\Phi}_{k}=C(N, d, s) \frac{1}{N_{Q}^{d}} \sum_{j \in \mathcal{I}_{2 N N_{Q}}^{\prime}}\left|\frac{j}{N_{Q}}\right|^{2 s} Y_{d}\left(\frac{\pi}{N}\left(\frac{j}{N_{Q}}-k\right)\right) .
$$

We then calculate the DFT of $\tilde{\Phi}$ and obtain

$$
\begin{aligned}
\hat{\tilde{\Phi}}_{k} & =\frac{(2 \pi)^{2 s}}{S^{2 s} N S} \sum_{\kappa \in \mathcal{I}^{\prime}} \tilde{\Phi}_{\kappa} \mathrm{e}^{-\mathrm{i} \frac{2 \pi \kappa k}{2 N}} \\
& =\frac{(2 \pi)^{2 s}}{S^{2 s} N S^{d}} \sum_{\kappa \in \mathcal{I}^{\prime d}}\left(\sum_{j \in \mathcal{I}^{\prime}{ }_{N S}}|j|^{2 s} \mathrm{e}^{\mathrm{i} \frac{2 \pi j \kappa}{S N}}\right) \mathrm{e}^{-\mathrm{i} \frac{2 \pi \kappa k}{2 N}} \\
& =\frac{(2 \pi)^{2 s}}{S^{2 s} N S^{d}} \sum_{j \in \mathcal{I}^{\prime}{ }_{N S}^{d}}|j|^{2 s} Y\left(2 \pi\left(\frac{j}{S N}-\frac{k}{2 N}\right)\right) \\
& =\frac{\pi^{2 s}}{(2 N)^{d}} \frac{1}{N_{Q}^{d}} \sum_{j \in \mathcal{I}^{\prime d}{ }_{2 N N_{Q}}}\left|\frac{j}{N_{Q}}\right|^{2 s} Y\left(\frac{\pi}{N}\left(\frac{j}{N_{Q}}-k\right)\right),
\end{aligned}
$$

which completes the proof. 
3.4. Solving the Dirichlet problem. In section 2.1, we have seen how to implement the application of the discrete operator $\Phi^{N}$ to a vector $\mathbf{u} \in \mathbb{R}^{N^{d}}$ efficiently using the fast Fourier transformation algorithm. In this section, we will show how this is used to solve the fractional Poisson problem with Dirichlet exterior conditions, i.e., eq. (1.1), repeated here for convenience:

$$
\text { find } u \text { s.t. } \begin{cases}(-\Delta)^{s} u=f & \text { in } \Omega \\ u=0 & \text { in } \mathbb{R}^{d} \backslash \Omega\end{cases}
$$

where $\Omega \subset[0 ; 1)^{d} \subset \mathbb{R}^{d}$ is an arbitrary Lipschitz domain and $f$ is a given function. Originally, our methods operates on the full cube $[0 ; 1)^{d}$. This leads to the discretized problem

$$
\text { find } \mathbf{u} \in \mathbb{R}^{N^{d}} \text { s.t. } \Phi^{N} \mathbf{u}=\mathbf{f}
$$

where $\mathbf{f}=\left(f_{k}\right)_{k \in \mathcal{I}_{N}^{d}}, f_{k}=f\left(x_{k}\right), x_{k}=k / N$ and $\Phi^{N}$ is the discrete operator from eq. (2.6). As shown, the application of $\Phi^{N}$ to a vector $\mathbf{u} \in \mathbb{R}^{N^{d}}$ can be implemented efficiently using the fast Fourier transformation algorithm. It is thus feasible to solve eq. (3.25) using iterative methods that work through subsequent applications of the operator instead of inverting them directly. In the present case, we use the conjugate gradient method [HS52] as it is fast, easy to implement and numerically stable. The same procedure has been used by Duo and Zhang in [DZ19] to solve the fractional Poisson problem in two-and three dimensions on rectangular (or cuboid) domains using their finite difference method, and by Minden and Ying [MY20] to solve the discrete system they obtain using singularity subtraction. To overcome the issue of being restricted to the cube $\Omega=[0 ; 1)^{d}$ and solve problems on arbitrary domains $\Omega \subset[0 ; 1)^{d}$, we embed the domain into the cube and set the coefficients outside of $\Omega$ to 0 . To implement this, we introduce a linear mapping

$$
S_{\Omega}: \mathbb{R}^{N^{d}} \longrightarrow \mathbb{R}^{N^{d}}
$$

such that for all $\mathbf{u}=\left(u_{k}\right)_{k \in \mathcal{I}_{N}^{d}} \in \mathbb{R}^{N^{d}}$

$$
\left(S_{\Omega} \mathbf{u}\right)_{k}= \begin{cases}u_{k} & \text { if } k / N \in \Omega \\ 0 & \text { otherwise }\end{cases}
$$

holds. Additionally, we define $S_{D}:=\mathbf{1}-S_{\Omega}$ where $\mathbf{1}$ is the identity on $\mathbb{R}^{N^{d}}$. Now, as we only want to solve eq. (3.25) for the indices that belong to $\Omega$, we solve the modified problem

$$
\text { find } \mathbf{u} \in \mathbb{R}^{N^{d}} \text { s.t. }\left\{\begin{array}{l}
S_{\Omega} \Phi^{N} S_{\Omega}^{T} \mathbf{u}=S_{\Omega} \mathbf{f} \\
S_{D} u=0
\end{array}\right.
$$

instead. Note that this is not a system on $\mathbb{R}^{N^{d}}$ anymore, but only on the subspace spanned by the indices that are selected by $S_{\Omega}$. The calculations are still done on the full $\mathbb{R}^{N^{d}}$ as this is the space where we can apply $\Phi^{N}$ efficiently, which is the prerequisite for using the conjugate gradient method. To show the capabilities of the methods, we apply it benchmark problems and problems arising from applications.

\section{Numerical EXPERIMENTS}

We present four numerical examples to demonstrate the efficiency of our implementation. First, we compare the scaled periodic fractional Laplacian (see section 3.1) to the sinc-fractional Laplacian for different scaling factors. Section 4.2 provides an experimental error analysis for a case where analytic solutions of the Dirichlet problem are known explicitly. As an example for the importance 
of using the correct exterior value conditions, we compare the fractional Allen-Cahn evolution equation for the periodically extendend and the Dirichlet case in section 4.4. Finally, in section 4.5, we show an application to image denoising as introduced in $[\mathrm{AB} 17 \mathrm{~b}]$.

4.1. Quadrature rules for the convolution kernel. In this section, we want to numerically evaluate how different quadrature rules in the calculation of the convolution kernel behave. For that reason, we calculate the discrete scaled periodic Fractional Laplacian $(\widetilde{-\Delta})_{S, N}^{s}$ of a function $u$ with different scaling factors $S$ and compare it to the sinc-fractional Laplacian $(-\Delta)_{N}^{s}$ of $u$ computed using different quadrature rules in the integration in eq. (3.22).

We obtain the simple error estimate

$$
\left\|(-\Delta)^{s} u-(-\Delta)_{N}^{s} u\right\| \leq\left\|(-\Delta)^{s} u-(\widetilde{(-\Delta})_{S, N}^{s} u\right\|+\left\|(\widetilde{(-\Delta})_{S, N}^{s} u-(-\Delta)_{N}^{s} u\right\|
$$

From lemma 3.1, we know that the first term is $\mathcal{O}\left(S^{-d+2 s}\right)$, and the second term can be calculated using the methods presented in sections $2.2,3.1$ and 4.1 .

In order to obtain good accuracy for calculating the sinc-fractional Laplacian at a reasonable computational cost, we aim to use better quadrature rules than the naive one presented in eq. (3.23). Basically, we can use any quadrature rule, but for efficient implementation, we should use the same quadrature rule on each of the $d$-dimensional cubes $\left[j_{1} ; j_{1}+1\right] \times \cdots \times\left[j_{d} ; j_{d}+1\right]$. This causes some issues as some cubes contain an integrand with a singularity due to the factor $|j+\omega|^{2 s}$ if $j_{i} \in\{-1,0\}$ for some $i \in\{1, \ldots, d\}$. Nevertheless, we obtain good results using a Gauß-Legendre quadrature rule. The results are illustrated in fig. 1, where we show the second term in the estimate eq. (4.1). Due to theorem 3.1, a sinc-fractional Laplacian with exactly computed convolution kernel corresponds to a periodic fractional Laplacian with 'infinite' scaling. In that case the difference between the sinc-fractional Laplacian and $\widetilde{(-\Delta})_{S, N}^{s}$ would decrease at the rate $\mathcal{O}\left(S^{-d+2 s}\right)$. In the figure, one can see that this rate applies until the quadrature errors from the computation of the convolution kernel dominate. Naturally, this happens later for more exact quadratures. In the numerical experiments below, we thus employ an $n^{d}$-point tensor product Gauß-Legendre quadrature, with $n=7$ for $d=2$ and $n=5$ for $d=3$. fig. 1 shows that a 7-point Gauß-Legendre quadrature corresponds roughly to a scale factor $S$ between 40 and 60, depending on $s$.

4.2. Function with constant fractional Laplacian on the unit sphere. One of the few examples where the solution to the fractional Laplace Dirichlet problem is known explicitly is the problem

$$
\text { find } u \text { s.t. } \begin{cases}(-\Delta)^{s} u=1 & \text { in } \Omega \\ u=0 & \text { in } \mathbb{R}^{d} \backslash \Omega .\end{cases}
$$

For $\Omega=\left\{x \in \mathbb{R}^{d}|| x \mid<1\right\}$, the solution to eq. (4.2) is given by (see [BV16])

$$
u=C_{u}(d, s) \max \left\{0,\left(1-|x|^{2}\right)\right\}^{s}
$$

where $C_{u}(d, s)=\Gamma(d / 2) \cdot\left(2^{2 s} \Gamma(d / 2+s) \Gamma(1+s)\right)^{-1}$. After shifting and scaling the problem to a disc or a sphere that is a subset of the cube $[0 ; 1)^{d}$, and using the method from section 3.4, we obtain the results shown in fig. 3 in the case $d=2$, which clearly resemble the expected results. In table 1 , we show the number of iterations of the conjugate gradient method required until the residual $\|\mathbf{r}\|_{L^{2}}=\frac{1}{N^{d}} \sum_{k \in \mathcal{I}_{N}^{d}} r_{k}^{2}$ dropped below $10^{-8}$ and in table 2 , we show how the number of iterations scales w.r.t $N$. Since the conditioning of the problem becomes worse for finer grid resolution, it is expected that more iterations are necessary for increasing $N$. We note that for lower fractional 


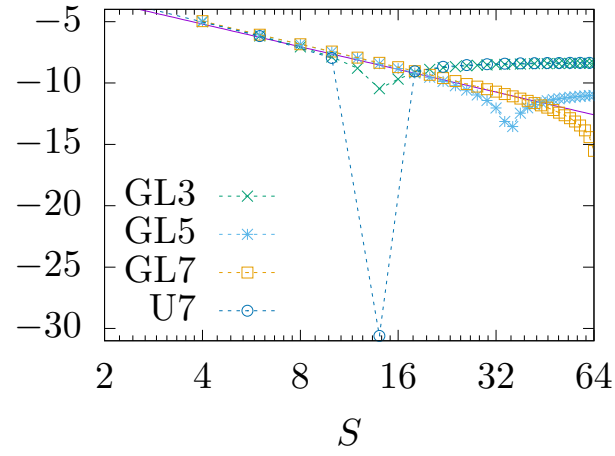

(A) $s=1 / 3$

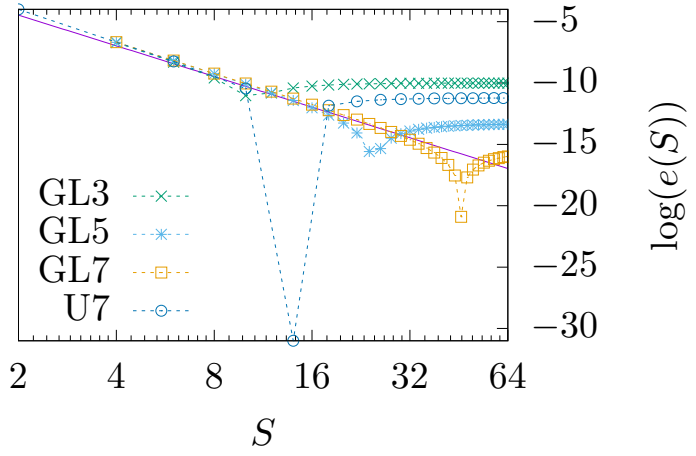

(B) $s=2 / 3$

Figure 1. Comparison of the scaled periodic fractional Laplacian and our implementation of the sinc-fractional Laplacian applied to a standard mollifier with support in $[0 ; 1)^{2}$ for fixed $N=64$. The difference is measured as $e(S)=$ $\left\|(-\Delta)_{N}^{s} u-(\overline{-\Delta})_{N, S}^{s} u\right\|_{\ell \infty}$. The solid purple line shows the rate $\mathcal{O}\left(S^{-d+2 s}\right)$. GL3, GL5 and GL7 denote Gauss-Legendre integration with 3,5 and 7 points in each spatial direction, U7 denotes 7 uniformly spaced quadrature points in each spatial direction. We see that at $S=2 N_{Q}=14$, the difference $e(S)$ is practically zero for this quadratue rule. This illustrates theorem 3.2.

TABle 1. Number of CG-iterations needed to solve the discretized linear system. The total number of Degrees of Freedom (DoFs) is $\mathcal{O}\left(N^{d}\right)$.

\begin{tabular}{|c|c|c|c|c|c|c|c|}
\hline & $N$ & $s=1 / 4$ & $s=1 / 3$ & $s=1 / 2$ & $s=2 / 3$ & $s=3 / 4$ & $s=1$ \\
\hline \multirow{10}{*}{$d=2$} & 8 & 8 & 8 & 8 & 8 & 8 & 8 \\
\hline & 16 & 14 & 17 & 21 & 24 & 26 & 27 \\
\hline & 32 & 19 & 24 & 34 & 45 & 51 & 63 \\
\hline & 64 & 25 & 32 & 48 & 75 & 91 & 132 \\
\hline & 128 & 31 & 43 & 76 & 127 & 161 & 271 \\
\hline & 256 & 40 & 57 & 112 & 208 & 281 & 545 \\
\hline & 512 & 50 & 75 & 163 & 340 & 488 & 1089 \\
\hline & 1024 & 61 & 97 & 234 & 550 & 882 & 2056 \\
\hline & 2048 & 76 & 127 & 343 & 1021 & 1660 & 4257 \\
\hline & 4096 & 94 & 166 & 535 & 1668 & 3010 & 8406 \\
\hline \multirow{6}{*}{$d=3$} & 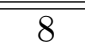 & $\bar{~} 10$ & $\overline{11}$ & $\overline{12}$ & $\bar{~} 13$ & $\overline{\overline{13}}$ & $\overline{\overline{13}}$ \\
\hline & 16 & 14 & 16 & 22 & 28 & 31 & 41 \\
\hline & 32 & 18 & 23 & 33 & 48 & 57 & 92 \\
\hline & 64 & 23 & 30 & 49 & 79 & 101 & 189 \\
\hline & 128 & 29 & 40 & 73 & 128 & 174 & 386 \\
\hline & 256 & 36 & 53 & 107 & 211 & 299 & 795 \\
\hline
\end{tabular}

exponent $s$, the required number of iterations is lower - this is reasonable as the largest eigenvalue of the sinc-fractional Laplacian should scale like $N^{2 s}$. 
TABLE 2. Scaling exponent $\beta$ of the number $N^{\beta}$ of CG-iterations depending on grid size $N$.

\begin{tabular}{|rr|c|c|c|c|c|c|}
\hline & $s$ & $1 / 4$ & $1 / 3$ & $1 / 2$ & $2 / 3$ & $3 / 4$ & 1 \\
\hline \multirow{2}{*}{$\beta$} & $d=2$ & 0.27 & 0.36 & 0.55 & 0.71 & 0.76 & 1.02 \\
\cline { 2 - 8 } & $d=3$ & 0.32 & 0.41 & 0.56 & 0.71 & 0.78 & 1.04 \\
\hline
\end{tabular}

TABLE 3. Time (in seconds) needed to solve the discretized system. These are the actual times it took to compute the results used for fig. 2.

\begin{tabular}{|c|c|c|c|c|c|c|c|c|}
\hline & $N$ & \#DoF & $s=1 / 4$ & $s=1 / 3$ & $s=1 / 2$ & $s=2 / 3$ & $s=3 / 4$ & $s=1$ \\
\hline \multirow{8}{*}{$d=2$} & 32 & 651 & $1.0 \mathrm{e}-3$ & $1.0 \mathrm{e}-3$ & $2.0 e-3$ & $2.0 \mathrm{e}-3$ & $3.0 \mathrm{e}-3$ & $4.0 \mathrm{e}-3$ \\
\hline & 64 & 2,605 & $4.0 \mathrm{e}-3$ & $5.0 \mathrm{e}-3$ & $7.0 \mathrm{e}-3$ & $1.2 \mathrm{e}-2$ & $1.3 \mathrm{e}-2$ & $2.7 \mathrm{e}-2$ \\
\hline & 128 & 10,423 & $1.4 \mathrm{e}-2$ & $1.8 \mathrm{e}-2$ & $3.2 \mathrm{e}-2$ & $5.3 e-2$ & $7.2 \mathrm{e}-2$ & $1.5 \mathrm{e}-1$ \\
\hline & 256 & 41,692 & $5.1 \mathrm{e}-2$ & $7.3 e-2$ & $1.4 \mathrm{e}-1$ & $2.8 \mathrm{e}-1$ & $3.8 \mathrm{e}-1$ & $1.0 \mathrm{e}+0$ \\
\hline & 512 & 166,768 & $3.8 \mathrm{e}-1$ & $5.6 \mathrm{e}-1$ & $1.2 \mathrm{e}+0$ & $2.6 \mathrm{e}+0$ & $3.8 \mathrm{e}+0$ & $1.2 \mathrm{e}+1$ \\
\hline & 1024 & 667,075 & $1.9 \mathrm{e}+0$ & $3.0 \mathrm{e}+0$ & $7.0 \mathrm{e}+0$ & $1.7 \mathrm{e}+1$ & $2.7 \mathrm{e}+1$ & $1.0 \mathrm{e}+2$ \\
\hline & 2048 & $2,668,300$ & $1.1 \mathrm{e}+1$ & $1.8 \mathrm{e}+1$ & $4.4 \mathrm{e}+1$ & $1.2 \mathrm{e}+2$ & $1.9 \mathrm{e}+2$ & $8.5 e+2$ \\
\hline & 4096 & $10,673,203$ & $5.8 \mathrm{e}+1$ & $1.0 \mathrm{e}+2$ & $3.2 \mathrm{e}+2$ & $9.8 \mathrm{e}+2$ & $1.7 \mathrm{e}+3$ & $8.7 e+3$ \\
\hline \multirow{5}{*}{$d=3$} & 16 & 1,563 & $7.0 \mathrm{e}-3$ & $8.0 \mathrm{e}-3$ & $1.0-2$ & $1.3 \mathrm{e}-2$ & $1.5-2$ & $2.0 \mathrm{e}-2$ \\
\hline & 32 & 12,507 & $9.9 \mathrm{e}-2$ & $1.3 \mathrm{e}-1$ & $1.8-1$ & $2.6 \mathrm{e}-1$ & $3.1-1$ & $5.3 e-1$ \\
\hline & 64 & 100,061 & $1.4 \mathrm{e}+0$ & $1.8 \mathrm{e}+0$ & $3.0+0$ & $4.8 \mathrm{e}+0$ & $6.0+0$ & $1.1 \mathrm{e}+1$ \\
\hline & 128 & 800,490 & $1.9 \mathrm{e}+1$ & $2.6 \mathrm{e}+1$ & $4.8+1$ & $8.1 e+1$ & $1.1+2$ & $2.5 \mathrm{e}+2$ \\
\hline & 256 & $6,403,922$ & $2.0 \mathrm{e}+2$ & $3.0 \mathrm{e}+2$ & $6.9+2$ & $1.3 \mathrm{e}+3$ & $2.1+3$ & $5.5 e+3$ \\
\hline
\end{tabular}

In table 3, we present the time that was required solve the system. The time grows when $s$ grows, reflecting the fact that we need more CG-iterations in this case. We implemented the algorithms in C++ using the FFTW library [FJ05] and the experiments where run on a standard office computer (6-core Intel Core i5-9500, 3.00 Ghz). In addition to the time that is needed to actually solve the system, one has to setup the operator $\Phi^{N}$. This convolution kernel has to be computed only once for each $d, s$ and mesh size $N$. It is independent of the domain $\Omega \subset[0,1)^{d}$. Especially for large values of $s$, this time is small compared to the time that is needed to actually solve the system. We provide the details in table 4 .

Figure 4 shows the results for $d=3$. In this case, we used only a 5-point Gauss-Legendre rule in order to reduce computation time.

As a numerical analysis of our method is still pending and remains part of future work, we experimentally evaluate the capabilities of our method. For that, we solve problem eq. (4.2) for different values of $s$ on grids of increasing size $N^{d}$ for $d=2$ and $d=3$. We approximate the $L_{2}$-error as

$$
\left\|u_{N}-u\right\|_{2} \approx \sqrt{\frac{1}{N^{d}} \sum_{k}\left(u_{k}-u\left(x_{k}\right)\right)^{2}},
$$

where $u_{N}=\sum_{k \in \mathcal{I}_{N}^{d}} u_{k} \varphi_{k}^{N}$ is the solution computed using the sinc-fractional Laplacian and $u$ is the known analytic solution. The results can be seen in fig. 2 (left) for the $2 d$-case and in fig. 2 (right) for 
TABle 4. Time (in seconds) needed to setup $\Phi^{N}$ in $d=2$ and $d=3$ dimensions using a $7^{2}(d=2)$ or $5^{3}(d=3)$ point Gauß-Legendre quadrature. Notice that $\Phi^{N}$ is applied to a vector of size $N^{d}$ and that $\Phi^{N}$ has $(2 N)^{d}$ entries

\begin{tabular}{|c|c|c|c|c|c|c|c|}
\hline & $N$ & $s=1 / 4$ & $s=1 / 3$ & $s=1 / 2$ & $s=2 / 3$ & $s=3 / 4$ & $s=1$ \\
\hline \multirow{10}{*}{$d=2$} & $\overline{8}$ & $\overline{c 1.4 \mathrm{e}-2}$ & $\overline{1.5 \mathrm{e}-2}$ & $\overline{1.4 \mathrm{e}-2}$ & $\overline{1.5 \mathrm{e}-2}$ & $\overline{1.5 \mathrm{e}-2}$ & $1.4 \mathrm{e}-2$ \\
\hline & 16 & $5.2 \mathrm{e}-2$ & $5.1 \mathrm{e}-2$ & $5.1 \mathrm{e}-2$ & $5.2 \mathrm{e}-2$ & $5.2 \mathrm{e}-2$ & $5.0 \mathrm{e}-2$ \\
\hline & 32 & $1.1 \mathrm{e}-1$ & $1.1 \mathrm{e}-1$ & $9.7 \mathrm{e}-2$ & $1.1 \mathrm{e}-1$ & $1.1 \mathrm{e}-1$ & $9.8 \mathrm{e}-2$ \\
\hline & 64 & $3.1 \mathrm{e}-1$ & $3.0 \mathrm{e}-1$ & $2.7 \mathrm{e}-1$ & $3.0 \mathrm{e}-1$ & $3.0 \mathrm{e}-1$ & $2.7 \mathrm{e}-1$ \\
\hline & 128 & $9.5 \mathrm{e}-1$ & $9.5 \mathrm{e}-1$ & $8.2 \mathrm{e}-1$ & $9.5 \mathrm{e}-1$ & $9.5 \mathrm{e}-1$ & $8.2 \mathrm{e}-1$ \\
\hline & 256 & $3.4 \mathrm{e}+0$ & $3.4 \mathrm{e}+0$ & $2.9 \mathrm{e}+0$ & $3.4 \mathrm{e}+0$ & $3.4 \mathrm{e}+0$ & $2.9 \mathrm{e}+0$ \\
\hline & 512 & $1.3 \mathrm{e}+1$ & $1.3 \mathrm{e}+1$ & $1.1 \mathrm{e}+1$ & $1.3 \mathrm{e}+1$ & $1.3 \mathrm{e}+1$ & $1.1 \mathrm{e}+1$ \\
\hline & 1024 & $5.2 \mathrm{e}+1$ & $5.2 \mathrm{e}+1$ & $4.3 \mathrm{e}+1$ & $5.2 \mathrm{e}+1$ & $5.2 \mathrm{e}+1$ & $4.3 \mathrm{e}+1$ \\
\hline & 2048 & $2.1 \mathrm{e}+2$ & $2.1 \mathrm{e}+2$ & $1.7 \mathrm{e}+2$ & $2.1 \mathrm{e}+2$ & $2.1 \mathrm{e}+2$ & $1.7 \mathrm{e}+2$ \\
\hline & 4096 & $8.2 \mathrm{e}+2$ & $8.2 \mathrm{e}+2$ & $6.9 \mathrm{e}+2$ & $8.2 \mathrm{e}+2$ & $8.2 \mathrm{e}+2$ & $6.9 \mathrm{e}+2$ \\
\hline \multirow{6}{*}{$d=3$} & $\overline{88}$ & $\overline{1.8 \mathrm{e}-1}$ & $\overline{1.8 \mathrm{e}-1}$ & $\overline{1.5 \mathrm{e}-1}$ & $\overline{1.8 \mathrm{e}-1}$ & $\overline{1.8 \mathrm{e}-1}$ & 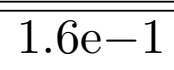 \\
\hline & 16 & $1.4 \mathrm{e}+0$ & $1.4 \mathrm{e}+0$ & $1.2 \mathrm{e}+0$ & $1.4 \mathrm{e}+0$ & $1.4 \mathrm{e}+0$ & $1.2 \mathrm{e}+0$ \\
\hline & 32 & $1.1 \mathrm{e}+1$ & $1.1 \mathrm{e}+1$ & $9.4 \mathrm{e}+0$ & $1.1 \mathrm{e}+1$ & $1.1 \mathrm{e}+1$ & $9.4 \mathrm{e}+0$ \\
\hline & 64 & $8.7 e+1$ & $8.7 \mathrm{e}+1$ & $7.6 \mathrm{e}+1$ & $8.6 \mathrm{e}+1$ & $8.7 \mathrm{e}+1$ & $7.6 \mathrm{e}+1$ \\
\hline & 128 & $7.1 \mathrm{e}+2$ & $7.1 \mathrm{e}+2$ & $6.2 \mathrm{e}+2$ & $7.1 \mathrm{e}+2$ & $7.1 \mathrm{e}+2$ & $6.2 \mathrm{e}+2$ \\
\hline & 256 & $5.7 \mathrm{e}+3$ & $5.7 \mathrm{e}+3$ & $5.0 \mathrm{e}+3$ & $5.7 \mathrm{e}+3$ & $5.7 \mathrm{e}+3$ & $5.0 \mathrm{e}+3$ \\
\hline
\end{tabular}
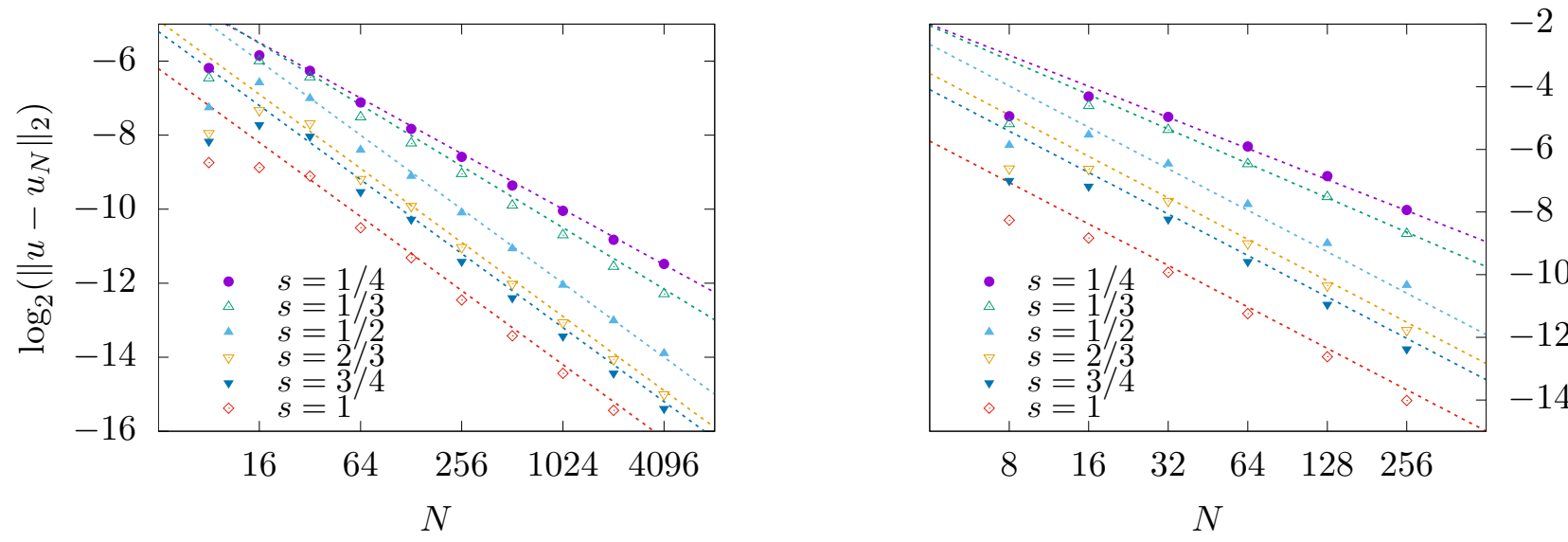

FIgURE 2. Experimental convergence analysis as a log-log plot in 2 dimensions (left) and 3 dimensions (right). The decay conforms to the rates predicted in [AB17a, BDPM18, AG18], displayed as dashed lines in the plots. $N$ is the number of grid points in each direction, i.e., the total number of grid points is $N^{2}$ and $N^{3}$ in respectively. 
the $3 d$-case. We experimentally obtain the convergence rates shown in table 5 . In [AB17a], Acosta and Borthagaray proved for their finite element implementation the convergence rate $\mathcal{O}\left(h^{1-\varepsilon}\right)$ for mesh size $h=1 / N$ in the $H^{s}(\Omega)$-norm under appropriate smoothness assumptions on the domain $\Omega$. Using an Aubin-Nitsche argument, the convergence in the $L^{2}(\Omega)$-norm is $\mathcal{O}\left(h^{\min (1, s+1 / 2)}\right)$ [BDPM18, AG18], modulo $\varepsilon$ or a logarithmic correction. The rates that we obtain clearly recover this rate for $s<1$. Our method can also treat the case $s=1$, i.e., the standard Laplacian. Notice, however, the reduced rate in table 5 . This is due to the fact that the exact solution $u \notin H^{2}\left(\mathbb{R}^{d}\right)$.

TABLE 5. Experimentally determined convergence rates.

\begin{tabular}{|c|c|c|c|c|c|c|c|}
\hline & $s$ & $1 / 4$ & $1 / 3$ & $1 / 2$ & $2 / 3$ & $3 / 4$ & 1 \\
\hline \multirow{2}{*}{ Determined rate } & $d=2$ & 0.7329 & 0.8192 & 0.9622 & 1.0166 & 1.0189 & 1.0126 \\
\hline & $d=3$ & 0.7439 & 0.8324 & 0.9725 & 1.0360 & 1.0425 & 1.0306 \\
\hline Expected rate & & 0.75 & $0.8 \overline{3}$ & 1.00 & 1.00 & 1.00 & 1.00 \\
\hline
\end{tabular}

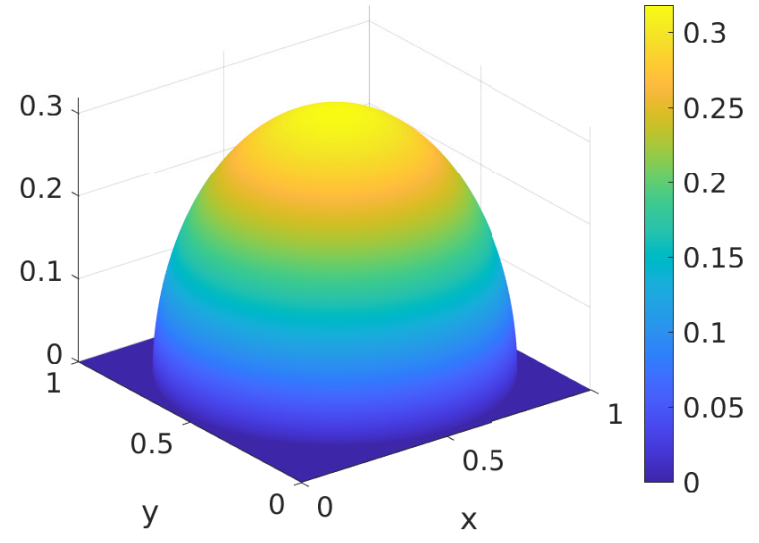

(A) $\mathbf{u}$

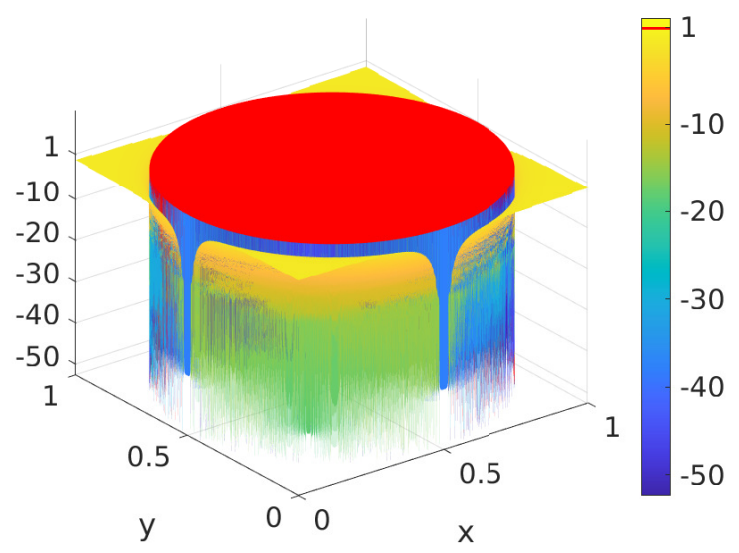

(B) $\Phi \mathbf{u}$

Figure 3. Discrete solution $\mathbf{u}(\mathrm{a})$ and $\Phi \mathbf{u}$ (b) for problem eq. (4.2) with $s=1 / 2$ using $2^{12} \times 2^{12}$ grid points. The solution clearly resembles the expected solution, given in eq. (4.3). In (b), values closer than $\varepsilon=10^{-5}$ to 1 are colored red to show that the sinc-fractional Laplacian is constant in the correct region.

4.3. Function with constant fractional Laplacian on an L-shaped domain. To show that we can treat domains other than the sphere, we solve the boundary value problem with constant right-hand side on an L-shaped domain. The results are provided in fig. 5 and visibly resemble the numerical solutions provided e.g. in $\left[\mathrm{LPG}^{+} 20\right]$.

If $\Omega$ is not the unit sphere anymore, then the solution to eq. (4.2) is not available analytically. Therefore, we perform a numerical error analysis where we compare the solution on a fine mesh with $N=2^{12}$ points in each spatial direction to solutions on coarser meshes. The results of our computations can bee seen in fig. 5 . We present the approximated $L^{2}$-errors in fig. 6 . 


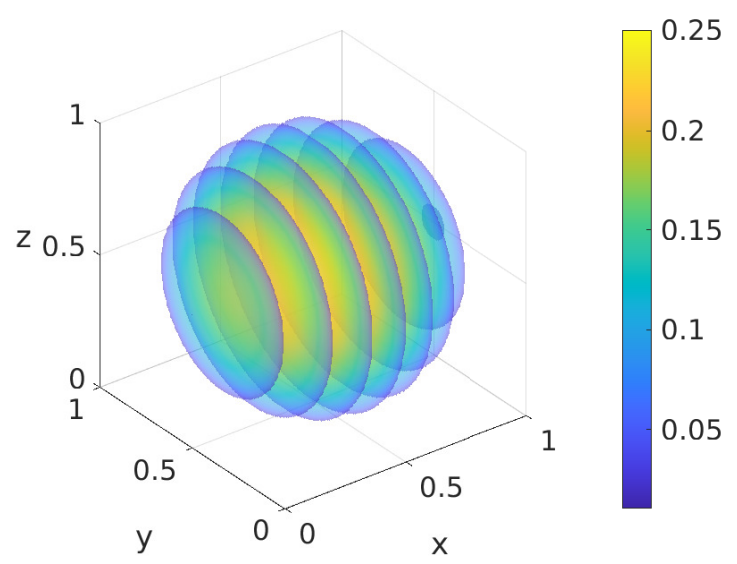

(A) $\mathbf{u}$



(B) $\Phi \mathbf{u}$

Figure 4. Discrete solution $\mathbf{u}$ (a) and $\Phi \mathbf{u}$ (b) for problem eq. (4.2) with $s=1 / 2$ using $\left(2^{8}\right)^{3}$ grid points. In (b), values closer than $\varepsilon=10^{-5}$ to 1 are colored red to show that the sinc-fractional Laplacian is constant in the correct region.

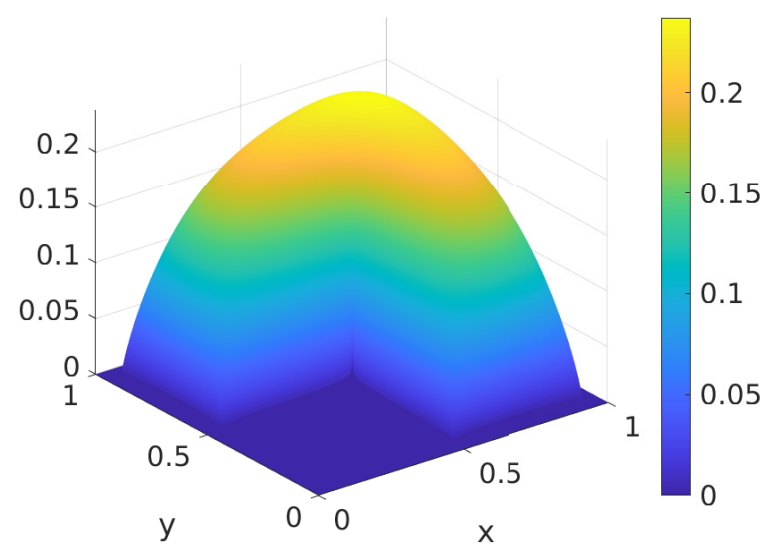

(A) $\mathbf{u}$

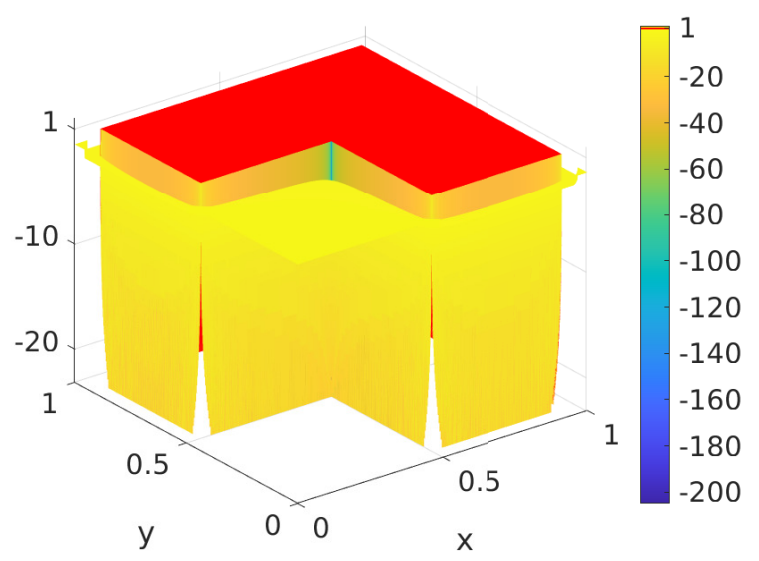

(B) $\Phi \mathbf{u}$

Figure 5. Discrete solution $\mathbf{u}(\mathrm{a})$ and $\Phi \mathbf{u}$ (b) for problem eq. (4.2) with $s=1 / 2$ on an L-shaped domain using $2^{12} \times 2^{12}$ grid points. In (b), values closer than $\varepsilon=10^{-5}$ to 1 are colored red to show that the sinc-fractional Laplacian is constant in the correct region. The fractional Laplacian exhibits a strong singularity at the inside corner.

4.4. Fractional Allen-Cahn equation. As a practical application that shows that our method correctly implements the Dirichlet exterior value conditions instead of periodic exterior value conditions, we calculate the evolution of the fractional Allen-Cahn equation

$$
\partial_{t} u+(-\Delta)^{\frac{1}{2}} u=-\frac{1}{\varepsilon} W^{\prime}(u)
$$




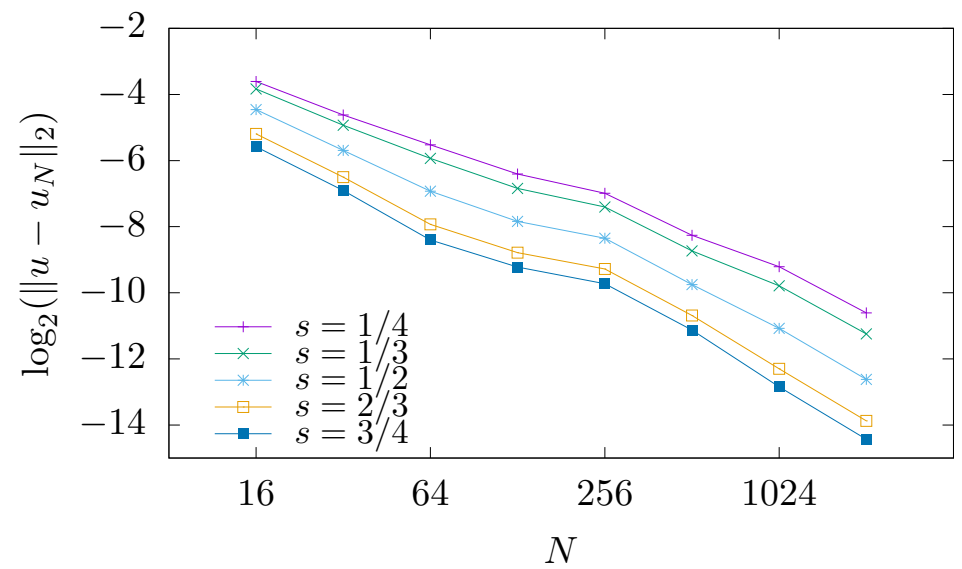

FigURE 6. Experimental convergence analysis for the boundary value problem on an L-shaped domain. The solution on the coarse grids $\left(N=2^{2}, \cdots, 2^{11}\right.$ in each spatial direction) where compared to the solution on a the finest grid $\left(N=2^{12}\right.$ in each spatial direction).

for $\varepsilon=2 \cdot 10^{-3}$; as an example here for fractional exponent $s=\frac{1}{2}$. The function $W: \mathbb{R} \rightarrow \mathbb{R}$ is a typical quartic double well potential of the form $W(u)=\frac{1}{4} u^{2}(u-1)^{2}$. It has recently been proved [ABS94, SV12] that, for $\varepsilon \rightarrow 0$, the associated energy $\frac{1}{\log \varepsilon}\left([u]_{H^{\frac{1}{2}}}^{2}+\frac{1}{\varepsilon} W(u)\right)$ converges (modulo constants) in the sense of $\Gamma$-convergence to the perimeter (in our one-dimensional case, a jump set counting functional). Heuristically, this energy prefers states of $u \in\{0,1\}$. The fractional Sobolev norm ensures that transitions between these two states can not take place arbitrarily rapidly in space.

The gradient flow, accelerated by a factor of $\frac{1}{\varepsilon \log \varepsilon}$ as computed here, converges in one spatial dimension to a kink-antikink annihilation-type dynamic [GM12]. Again, heuristically, two nearby states close to $u=+1$, separated by a gap where $u=0$, attract each other due to the long range interaction via the fractional operator, so the two phase transitions (or kinks) move closer to each other. The expected behavior in the $\varepsilon \rightarrow 0$ limit is a kink velocity proportional to the reciprocal of the distance to the antikink (and vice-versa).

Note that the evolution for small $\varepsilon>0$ is substantially faster than for the classical local Allen-Cahn equation, where exponentially slow kink-antikink annihilation was shown [BK90, CP89].

We discretize the equation in time using an implicit Euler scheme with time step $\tau$ and obtain

$$
\left(\mathbf{1}+\tau(-\Delta)^{\frac{1}{2}}\right) \mathbf{u}^{t+1}=-\frac{\tau}{\varepsilon} W^{\prime}\left(\mathbf{u}^{t}\right)+\mathbf{u}^{t} .
$$

To illustrate the differences due to exterior domain condition, we choose either the periodic fractional Laplacian or the sinc-fractional Laplacian and compare. In the first case, the system eq. (4.4) can be solved directly using the discrete Fourier transformation, see, e.g., [AB17b] for details. In the case of the sinc-fractional Laplacian, we use the conjugate gradient method as explained in section 3.4. We choose the domain $\Omega=[0 ; 1) \subset \mathbb{R}$ and $\mathbf{u}^{0}(x)=\chi_{[1 / 4 ; 3 / 4]}(x)$, i.e., the indicator function on the interval $\left[\frac{1}{4} ; \frac{3}{4}\right]$. In fig. 7 , we show the evolution for different times. It can be clearly seen that the solution for periodic conditions reaches a steady state, due to symmetry: the attraction of the 
kink and antikink at $x=\frac{1}{4}$ and $x=\frac{3}{4}$, respectively is balanced by the attraction to their periodic mirror images past the domain boundary. In the case of the true Dirichlet problem, the annihilation is clearly visible. Figure 8 shows the time-evolution of the total mass (i.e., $\left.m(t)=\int_{0}^{1} u(x, t) \mathrm{d} x\right)$ as well as the position of the left kink (or phase transition) over time. The fit to the solution of the aforementioned limit equation (which is of the form $a \sqrt{t_{0}-t}$ with parameters $a$ and $t_{0}$ ) is included on the left panel in fig. 8 .

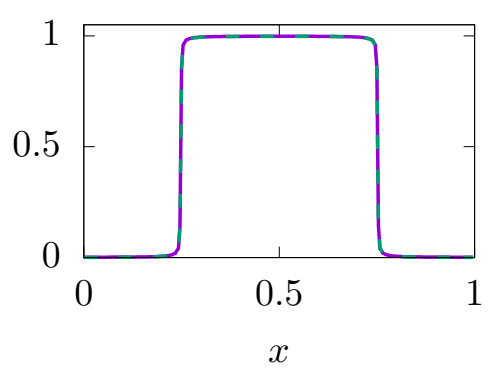

(A) $t=0.01$

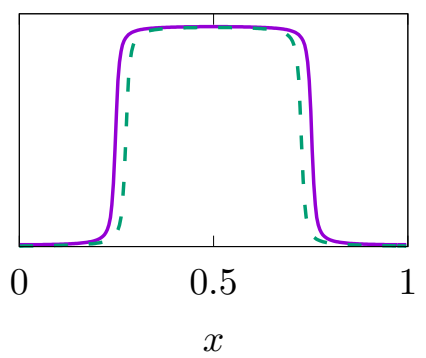

(B) $t=10$

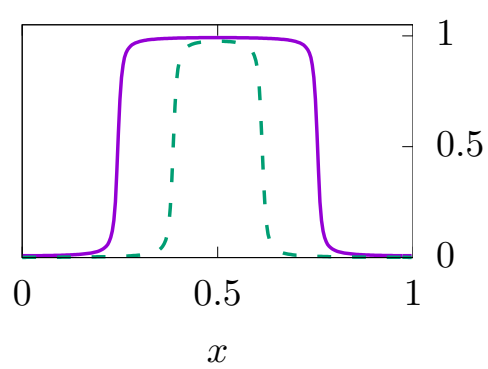

(C) $t=40$

Figure 7. The solution $u(x, t)$ of the fractional Allen-Cahn equation for different times $t$. The dashed geen line shows the evolution for the sinc-fractional Laplacian and the solid purple line the evolution for the periodic fractional Laplacian.
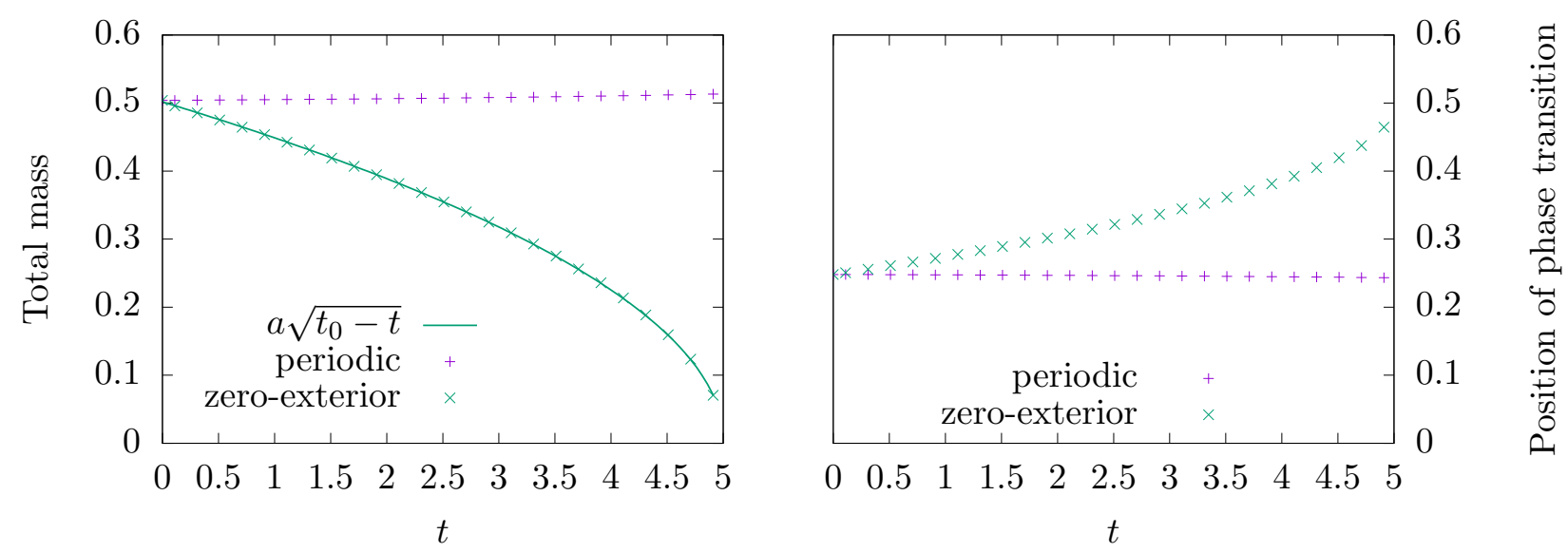

FiguRE 8. Evolution of the total mass (i.e., the integral of $u$ ) in the interval $[0 ; 1]$ (left) and of the position of the first phase transition (right) using both the periodic and the zero-exterior value Dirichlet (sinc)-fractional Laplacian for the fractional Allen-Cahn Equation. The continuous line in the left panel shows a fit to a squareroot function $a \sqrt{t_{0}-t}$, with $a=0.2242, t_{0}=5.0104$.

4.5. Image denoising. In $[\mathrm{AB} 17 \mathrm{~b}]$, Antil and Bartels have proposed to solve an image denoising variational problem. Given a noisy image $g$, it amounts to:

$$
\min _{u} \frac{1}{2} \int_{\Omega}\left|(-\Delta)^{\frac{s}{2}} u\right|^{2}+\frac{\alpha}{2} \int_{\Omega}|u-g|^{2}
$$


i.e., they use the fractional Laplacian as the regularizer. Here $\alpha>0$ is the regularization parameter. Starting from the seminal work of Rudin-Osher-Fatemi [ROF92], where they used the total variation as a regularizer, such variational models are being regularly used in imaging science. The key advantage of the fractional Laplacian regularizer from $[\mathrm{AB} 17 \mathrm{~b}]$ is the fact that one arrives at the following linear Euler-Lagrange equations:

$$
(-\Delta)^{s} u+\alpha(g-u)=0 .
$$

In the respective work, the authors use the spectral fractional Laplacian which applies periodic boundary conditions. In contrast, we use our method that uses Dirichlet-exterior value conditions. Here, we subtract the mean $\bar{g}$ of $g$ from $g$ before the calculations and add it back afterwards. The results can be seen in fig. 9. The goal of this example is not to further illustrate the effectiveness of fractional Laplacian as a regularizer, but to show that we can obtain comparable results using the approach considered in this paper. This clearly follows from our example.

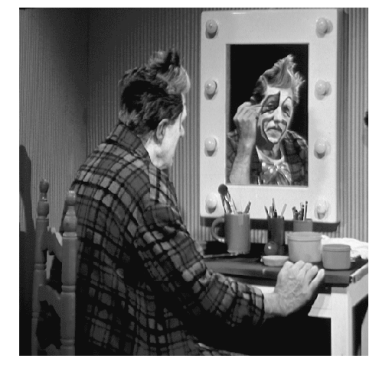

(A)

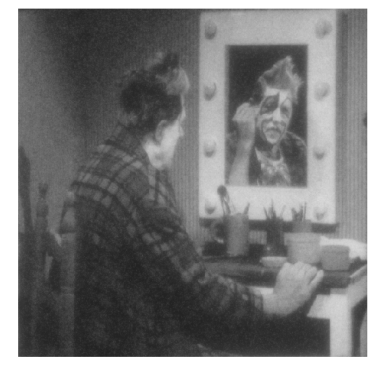

(C)



(B)



(D)

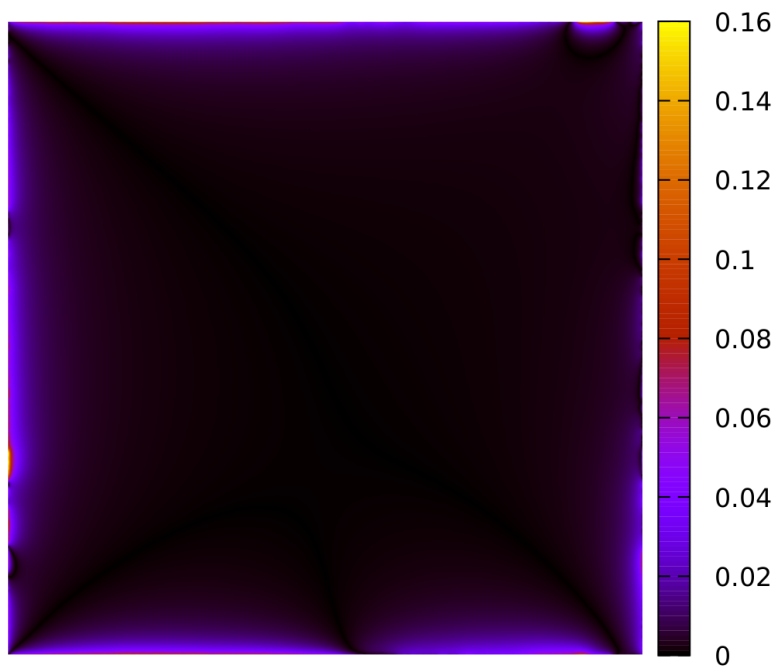

(E)

Figure 9. (a) Original image, (b) image corrupted with Gaussian noise, (c) denoised image using our method, and $(\mathrm{d})$ the image denoised with the spectral method from $[\mathrm{AB} 17 \mathrm{~b}]$. We chose $s=0.42$ and $\alpha=10 \cdot 2 \pi$. The difference of the image denoised with our method and denoised with the spectral method of [AB17b] (see fig. 9) is shown in (e). The differences clearly concentrate at the boundary of the images as one would expect.

\section{Conclusion and Future Work}

The paper introduces a novel spectral method which allows efficient application of the fractional Laplacian in $\mathcal{O}(N \log (N))$ operations as well as a solution algorithm for fractional partial differential equations with Dirichlet exterior conditions. The proposed method works in both $2 d$ and $3 d$. We 
have further shown the effectiveness of the method in two applications: a fractional Allen-Cahn equation and an image denoising problem. The method works on arbitrary domains, for instance we have done computations on the ever popular L-shape domain. One potential limitation of our approach is that we can work only on uniform grids, a higher number of unknowns maybe required especially when the solution is expected to have have singularities. Regardless, the application of our discrete operator still retains its $\mathcal{O}(N \log (N))$ complexity.

This work opens up new opportunities for problems where nonlocal operators such as fractional Laplacians appear, especially in $3 d$. There are a number of open questions which are a matter of current investigation. In particular, (i) How to extend the proposed method to other exterior conditions such as Neumann or Robin and how to handle nonzero exterior conditions; (ii) a complete numerical analysis of the proposed method is currently missing; (iii) we have applied the proposed method to both linear elliptic and nonlinear parabolic (Allen-Cahn) equations, it will also be interesting to carry out analysis in the nonlinear setting of Allen-Cahn; (iv) it will be interesting to apply the proposed method to equilibrium problems such as variational inequalities and PDE constrained optimization problems.

\section{ApPendix A. Implementation DETAILs AND COMPUTATIONAL COMPLEXity}

In this section, we provide more details on the implementation of the algorithms. The indices in the following computations will be chosen such that they fit the indices of usual FFT implementations. All our implementations are written in C++ and rely on the FFTW library [FJ05]. Computing the discrete solution of the Dirichlet-exterior value problem eq. (1.1) essentially consists of two steps,

- setup the discrete operator $\Phi^{N}$,

- solve the (discrete) system $S_{\Omega} \Phi^{N} S_{\Omega}^{T} \mathbf{u}=S_{\Omega} \mathbf{f}$.

Regarding the first step, we do not actually calculate $\Phi^{N}$, but its DFT $\hat{\Phi}^{N}$ following the procedure described in section 3.3. Further details are provided in algorithm 1. The arithmetic operations $*,+$ are meant component-wise if applied to arrays. The computationally most demanding part in algorithm 1 is the 3-fold execution of the FFT-algorithm in lines 10, 11 and 13 of algorithm 1, which are needed to compute the inner sum on the right-hand side of eq. (3.22), i.e., the convolution. These FFTs have to be executed for each of the quadrature points, i.e., $N_{Q}$-times and they are of size $(2 N)^{d}$. However, this is done for all the $k \in \mathcal{I}_{2 N}^{d}$ at a total cost of $\mathcal{O}\left((2 N)^{d} \log \left((2 N)^{d}\right)\right)$ which is still small compared to a naive implementation at cost $\mathcal{O}\left(\left((2 N)^{d}\right)^{2}\right)$.

For the second step, i.e., the solution of the fractional PDE, we use the conjugate gradient (CG) method. The method solves a linear system

$$
\mathbf{A u}=\mathbf{b}, \quad \mathbf{b} \in \mathbb{R}^{N}, \mathbf{A} \in \mathbb{R}^{N \times N} \text { invertible symmetric and positive-definite }
$$

via successive applications of the matrix $\mathbf{A}$ instead of solving it directly. Consequently, the algorithm is fast if the application of the operator $\mathbf{A}$ can be computed efficiently. This is the case in our setting, since we need to compute the application (convolution) of $\Phi^{N}$ to a vector $\mathbf{u}$. As mentioned above, we do so by using FFT based algorithms in order to reduce the computational complexity. We have to introduce some padding in order to apply the zero-padding convolution instead of circular convolution as the FFT based algorithm would normally do. The details are provided in algorithm 2 . 

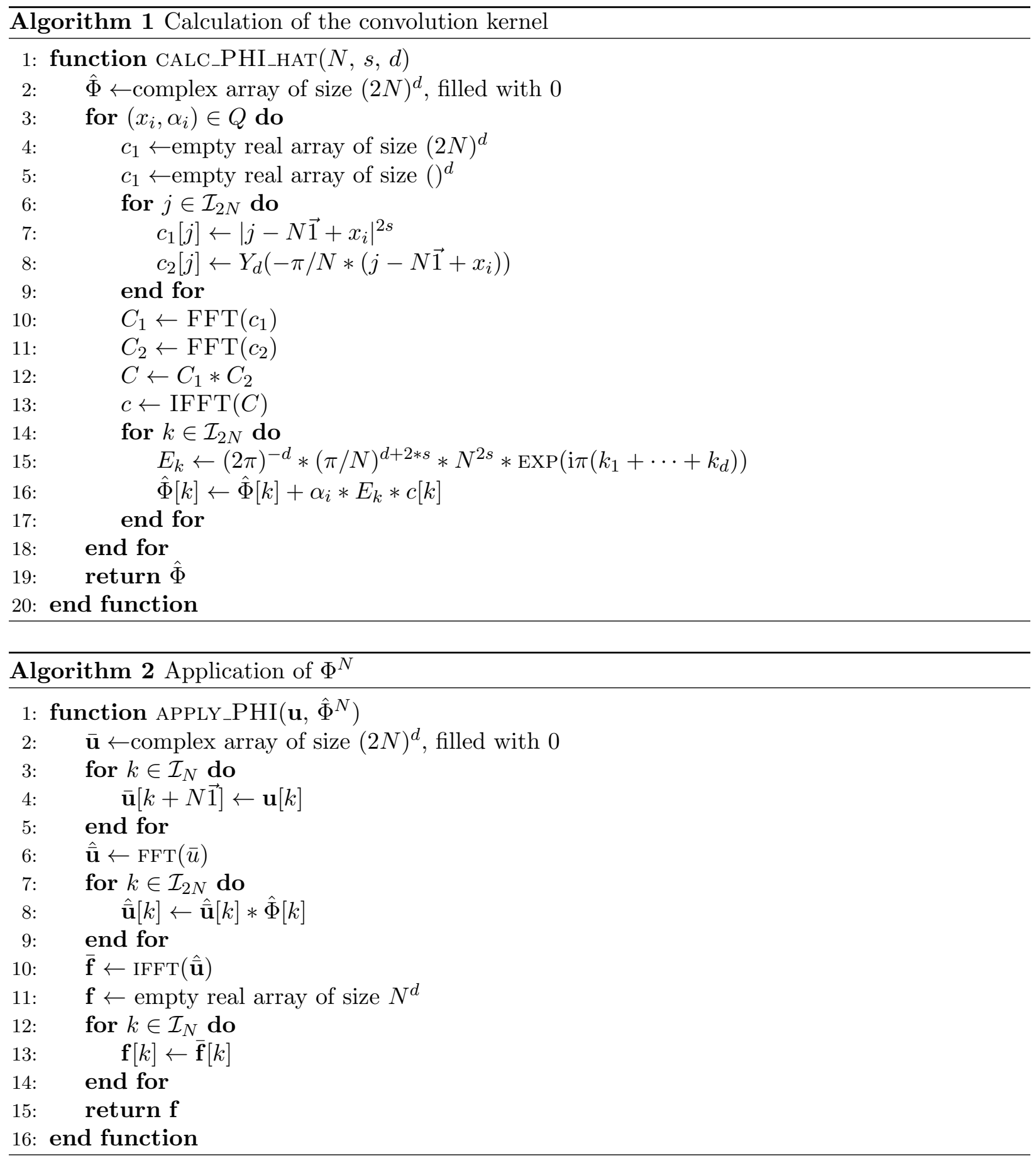

If the domain in the exterior value problem is the full cube $[0 ; 1)^{d}$, we can simply use the conjugate gradient method with the operator $\Phi^{N}$ using the efficient application described in algorithm 2 . If we want to restrict the exterior value problem to a domain $\Omega \subsetneq[0 ; 1)^{d}$, we use the strategy described in section 3.4 and further summarized in algorithm 3. Both the operator $\Phi^{N}$ and the operator restricted to a smaller area can be applied to a vector $\mathbf{u}$ at $\operatorname{cost} \mathcal{O}\left((2 N)^{d} \log \left((2 N)^{d}\right)\right)$ which is substantially less then the cost $\mathcal{O}\left(\left((2 N)^{d}\right)^{2}\right)$ of the naive implementation. 




\section{REFERENCES}

[AB17a] Gabriel Acosta and Juan Pablo Borthagaray. A fractional Laplace equation: regularity of solutions and finite element approximations. SIAM Journal on Numerical Analysis, 55(2):472-495, Jan 2017.

[AB17b] Harbir Antil and Sören Bartels. Spectral approximation of fractional PDEs in image processing and phase field modeling. Computational Methods in Applied Mathematics, 17, 042017.

[ABS94] Giovanni Alberti, Guy Bouchitté, and Pierre Seppecher. Un résultat de perturbations singulières avec la norme $H^{1 / 2}$. Comptes Rendus de l'Académie des Sciences. Série I. Mathématique, 319(4):333-338, 1994.

[ADK20] Harbir Antil, Zichao Wendy Di, and Ratna Khatri. Bilevel optimization, deep learning and fractional Laplacian regularizatin with applications in tomography. Inverse Problems, 36(6):064001, may 2020.

[AG18] Mark Ainsworth and Christian Glusa. Towards an efficient finite element method for the integral fractional Laplacian on polygonal domains, pages 17-57. Springer International Publishing, Cham, 2018.

[AKW19] Harbir Antil, Ratna Khatri, and Mahamadi Warma. External optimal control of nonlocal PDEs. Inverse Problems, 35(8):084003, 35, 2019.

[AM17] Mark Ainsworth and Zhiping Mao. Analysis and approximation of a fractional cahn-hilliard equation. SIAM Journal on Numerical Analysis, 55(4):1689-1718, 2017.

[AR19] H. Antil and C.N. Rautenberg. Sobolev spaces with non-Muckenhoupt weights, fractional elliptic operators, and applications. SIAM J. Math. Anal., 51(3):2479-2503, 2019.

[ASS15] Goro Akagi, Giulio Schimperna, and Antonio Segatti. Fractional cahn-hilliard, allen-cahn and porous medium equations. Journal of Differential Equations, 261, 022015.

[AV19] Nicola Abatangelo and Enrico Valdinoci. Getting acquainted with the fractional Laplacian. In Contemporary research in elliptic PDEs and related topics, volume 33 of Springer INdAM Ser., pages 1-105. Springer, Cham, 2019.

$\left[\mathrm{BBN}^{+} 18\right] \quad$ Andrea Bonito, Juan Pablo Borthagaray, Ricardo H. Nochetto, Enrique Otárola, and Abner J. Salgado. Numerical methods for fractional diffusion. Comput. Vis. Sci., 19(5-6):19-46, 2018.

[BDPM18] Juan Pablo Borthagaray, Leandro M. Del Pezzo, and Sandra Martinez. Finite element approximation for the fractional eigenvalue problem. Journal of Scientific Computing, 77(1):308-329, Oct 2018.

[BK90] L. Bronsard and R. V. Kohn. On the slowness of phase-boundary motion in one space dimension. Communications on Pure and Applied Mathematics, 43(8):983-997, December 1990.

[BLP19] Andrea Bonito, Wenyu Lei, and Joseph E. Pasciak. Numerical approximation of the integral fractional Laplacian. Numer. Math., 142(2):235-278, 2019. 
[BS11] Gerd Baumann and Frank Stenger. Fractional calculus and sinc methods. Fractional Calculus and Applied Analysis, 14(4), jan 2011.

[BV16] Claudia Bucur and Enrico Valdinoci. Nonlocal diffusion and applications. Springer, 2016.

[BWZ20] John Burkardt, Yixuan Wu, and Yanzhi Zhang. A unified meshfree pseudospectral method for solving both classical and fractional PDEs. arXiv e-prints, page arXiv:2009.10811, September 2020.

[Cat08] Carlo Cattani. Shannon wavelets theory. Math. Probl. Eng., pages Art. ID 164808, 24, 2008.

[Cat15] Carlo Cattani. Local fractional calculus on Shannon wavelet basis. In Fractional dynamics, pages 6-30. De Gruyter Open, Berlin, 2015.

[Cat18] Carlo Cattani. Sinc-fractional operator on Shannon wavelet space. Frontiers in Physics, 6:118, 2018.

[CP89] J. Carr and R. L. Pego. Metastable patterns in solutions of $u_{t}=\varepsilon 2 u_{x x}-f(u)$. Communications on Pure and Applied Mathematics, 42(5):523-576, 1989.

[CS06] Luis Caffarelli and Luis Silvestre. An extension problem related to the fractional Laplacian. Communications in Partial Differential Equations, 32, 082006.

[DNPV12] Eleonora Di Nezza, Giampiero Palatucci, and Enrico Valdinoci. Hitchhiker's guide to the fractional sobolev spaces. Bulletin des Sciences Mathématiques, 136:521-573, 072012.

[DZ19] Siwei Duo and Yanzhi Zhang. Accurate numerical methods for two and three dimensional integral fractional Laplacian with applications. Computer Methods in Applied Mechanics and Engineering, 355:639 - 662, 2019.

[FJ05] Matteo Frigo and Steven G. Johnson. The design and implementation of FFTW3. Proceedings of the IEEE, 93(2):216-231, 2005. Special issue on "Program Generation, Optimization, and Platform Adaptation".

[GM12] Maria del Mar González and Régis Monneau. Slow motion of particle systems as a limit of a reactiondiffusion equation with half-Laplacian in dimension one. Discrete and Continuous Dynamical Systems. Series A, 32(4):1255-1286, 2012.

[HO16] Yanghong Huang and Adam Oberman. Finite difference methods for fractional Laplacians. arXiv eprints, page arXiv:1611.00164, November 2016.

[HS52] M. R. Hestenes and E. Stiefel. Methods of conjugate gradients for solving linear systems. J Res NIST, 49(6):409-436, 1952.

[JLLZ15] Bangti Jin, Raytcho Lazarov, Yikan Liu, and Zhi Zhou. The galerkin finite element method for a multi-term time-fractional diffusion equation. Journal of Computational Physics, 281:825 - 843, 2015.

[Kwa15] Mateusz Kwaśnicki. Ten equivalent definitions of the fractional laplace operator. Fractional Calculus and Applied Analysis, 20, 072015.

$\left[\mathrm{LPG}^{+} 20\right] \quad$ Anna Lischke, Guofei Pang, Mamikon Gulian, Fangying Song, Christian Glusa, Xiaoning Zheng, Zhiping Mao, Wei Cai, Mark M. Meerschaert, Mark Ainsworth, and George Em Karniadakis. What is the fractional laplacian? a comparative review with new results. Journal of Computational Physics, 404:109009, 2020.

[MPSV18] Dominik Meidner, Johannes Pfefferer, Klemens Schürholz, and Boris Vexler. $h p$-finite elements for fractional diffusion. SIAM J. Numer. Anal., 56(4):2345-2374, 2018.

[MSW71] J. McNamee, F. Stenger, and E. L. Whitney. Whittaker's cardinal function in retrospect. Math. Comp., 25:141-154, 1971.

[MY20] Victor Minden and Lexing Ying. A simple solver for the fractional Laplacian in multiple dimensions. SIAM Journal on Scientific Computing, 42(2):A878-A900, 2020.

[NOS15] Ricardo H. Nochetto, Enrique Otárola, and Abner J. Salgado. A PDE approach to fractional diffusion in general domains: a priori error analysis. Found. Comput. Math., 15(3):733-791, 2015.

[ROF92] Leonid I. Rudin, Stanley Osher, and Emad Fatemi. Nonlinear total variation based noise removal algorithms. Physica D: Nonlinear Phenomena, 60(1):259 - 268, 1992.

[RRD19] Joel A. Rosenfeld, Spencer A. Rosenfeld, and Warren E. Dixon. A mesh-free pseudospectral approach to estimating the fractional laplacian via radial basis functions. Journal of Computational Physics, 390:306-322, 2019.

[Sil05] Luis Enrique Silvestre. Regularity of the obstacle problem for a fractional power of the Laplace operator. PhD thesis, The University of Texas at Austin, 72005.

[ST10] Pablo Raúl Stinga and José Luis Torrea. Extension problem and Harnack's inequality for some fractional operators. Communications in Partial Differential Equations, 35(11):2092-2122, 2010.

[Ste00] Frank Stenger. Summary of sinc numerical methods. Journal of Computational and Applied Mathematics, 121(1):379 - 420, 2000.

[Ste10] Frank Stenger. Handbook of Sinc Numerical Methods. CRC Press, Inc., USA, 2010. 
[SV12] Ovidiu Savin and Enrico Valdinoci. $\Gamma$-convergence for nonlocal phase transitions. Annales de l'Institut Henri Poincaré. Analyse Non Linéaire, 29(4):479-500, 2012.

[Val09] Enrico Valdinoci. From the long jump random walk to the fractional Laplacian. Bol. Soc. Esp. Mat. Apl. SeMA, (49):33-44, 2009.

[WvBWA20] C.J. Weiss, B.G. van Bloemen Waanders, and H. Antil. Fractional operators applied to geophysical electromagnetics. Geophysical Journal International, 220(2):1242-1259, 2020.

[XD18] Kailai Xu and Eric Darve. Spectral Method for the Fractional Laplacian in 2D and 3D. arXiv e-prints, page arXiv:1812.08325, December 2018.

Department of Mathematical Sciences and the Center for Mathematics and Artificial Intelligence (CMAI), George Mason University, Fairfax, VA 22030, USA.

Email address: hantil@gmu.edu

Abteilung für Angewandte Mathematik, Albert-Ludwigs-Universität Freiburg, Hermann-Herder-Strasse 10, 79104 Freiburg I. BR.

Email address: patrick.dondl@mathematik.uni-freiburg.de, ludwig.striet@mathematik.uni-freiburg.de 